\title{
THE MULTIMODAL CONSTITUTION OF OBJECTS: HOW CHURCH VIEWING IS ACCOMPLISHED
}

\author{
Reinhold Schmitt \\ Institute for the German Language, Mannheim, Germany
}

\begin{abstract}
Based on a single case analysis, this essay examines the question of what the structure of a church viewing is like. Within a theoretical framework which conceptualizes the church viewing as a cultural practice, "object constitution" is regarded as a central aspect and as a visitor's active achievement. For the composition of the church viewings corpus, visitors were not only filmed whilst walking through the church interior and visually perceiving certain aspects of the architecture, but were also asked to verbally comment their visual perceptions. The church viewing corpus was recorded with two cameras: an action camera, documenting from the church visitors' perspective; the other a contextual camera following the visitors on their way through the church interior.

This experimental design, which uses the verbal commenting of the visitors' own perception as a scientific method, allows reconstructing their church viewing concepts as a dynamic interplay of their visual selection und perception of the church interior and the verbal commenting of their visual perception.

The single case analysis proves that object constitution is in fact a visitor's active accomplishment by which parts of the church interior are brought forth in a new way: Anton, the analysed visitor, who examines in detail two large paintings hanging on the wall, constitutes them as "picture frames".

Key words: church viewing, cultural practice, constitution of objects, architecture-for-interaction, social topography, multimodal interaction analysis, interactionist space analysis, thinking aloud.

УДК 81-25:366.63

Дата поступления статьи: 14.11.2016

ББК 81.006

Дата принятия статьи: 15.12.2016
\end{abstract}

\section{МУЛЬТИМОДАЛЬНЫЙ ПОДХОД К ИЗУЧЕНИЮ «СОЗДАНИЯ ОБЬЕКТА»: КАК ОСУЩЕСТВЛЯЕТСЯ ПОСЕЩЕНИЕ ЦЕРКВИ}

\author{
Райнхольд Шмитт \\ Институт немецкого языка, г. Мангейм, Германия
}

\begin{abstract}
Аннотация. В статье на основе изучения конкретного случая представлено исследование вопроса о том, как структурирована практика посещения церкви.

В теоретическом плане, концептуализирующем посещение церкви как культурную практику, «создание объекта» рассматривается в качестве активной деятельности посещающего церковь.

В процессе создания корпуса практик посещения церкви осуществлялись видеозаписи посетителей во время их прохождения через пространство церкви и визуального восприятия определенных аспектов пространства, а также предлагалось вербально прокомментировать визуальное восприятие. При создании корпуса исследуемых практик применялись две видеокамеры: экшн-камера, которая документировала пространство восприятия посещающих церковь, и базовая камера видеонаблюдения, сопровождающая посетителей во время передвижения по пространству храма.

\footnotetext{
Э $\quad$ Экспериментальное исследование, в котором сознательно применяется метод «экзотетического гово구의 рения» (озвучивание мыслей вслух) в качестве научного метода, делает возможной реконструкцию концепта च посещения и представления его как динамической взаимосвязи визуального восприятия пространства церкви человеком и вербального сопровождения им его восприятия.

Представленный анализ конкретного случая показывает, что «создание объекта» является активной деятельностью, посредством которой посетитель церкви частично заново конструирует и означивает ее простран-
} ство.
\end{abstract}




\title{
ДискУССИи
}

Ключевые слова: посещение церкви, культурная практика, «создание объекта», интеракционная архитектура, социальная топография, мультимодальный анализ интеракции, интеракционный анализ пространства, экзотетическое говорение.

UDK 81-25:366.63

Eingereicht: 14.11.2016

BBK 81.006

Angenommen: 15.12.2016

\section{MULTIMODALE OBJEKTKONSTITUTION: WIE EINE KIRCHENBESICHTIGUNG FUNKTIONIERT}

\author{
Reinhold Schmitt \\ Institut für Deutsche Sprache, Mannheim, Deutschland
}

Abstract. In diesem Aufsatz wird einzelfallanalytisch der Frage nachgegangen, wie die Struktur einer Kirchenbesichtigung aussieht. Im theoretischen Rahmen, der die Kirchenbesichtigung als kulturelle Praktik konzeptualisiert, wird „Objektkonstitution“ als eine aktive Leistung des Kirchenbesichtigers in den Blick genommen. Bei den Aufnahmen zum Kirchenbesichtigungskorpus wurden die Besichtiger nicht nur bei ihrem Gang durch den Kirchenraum und der visuellen Wahrnehmung bestimmter Raumaspekte gefilmt. Sie wurden vielmehr darum gebeten, ihre visuelle Wahrnehmung durch begleitendes Sprechen auch zu kommentieren. Aufgezeichnet wurde das Besichtigungskorpus mit zwei Kameras: einer Actionkamera, die den Wahrnehmungsraum der Besichtiger dokumentiert, und einer Kontextkamera, die ihnen bei ihrem Weg durch den Raum folgt.

Dieses experimentelle Erhebungsdesign, bei dem exothetisches Sprechen bewusst als wissenschaftliche Erhebungsmethode eingesetzt wird, macht es möglich, das Besichtigungskonzept der Personen als dynamisches Zusammenspiel ihrer visuellen Wahrnehmung des Kirchenraums und ihrer wahrnehmungsbegleitenden Exothese zu rekonstruieren. Dass Objektkonstitution eine aktive Herstellung ist, durch die der Kirchenraum in den Relevanzen seines Betrachters teilweise neu entsteht, zeigt die Fallanalyse in exemplarischer Klarheit: Anton, der analysierte Besichtiger, der sich ausführlich mit zwei großen Gemälden beschäftigt, konstituiert diese de facto als „Bilderrahmen“, ohne überhaupt auf die dargestellten Szenen einzugehen.

Schlüsselwörter: Kirchenbesichtigung, kulturelle Praktik, Objektkonstitution, Interaktionsarchitektur, Sozialtopografie, multimodale Interaktionsanalyse, interaktionistische Raumanalyse, exothetisches Sprechen.

\section{Einleitung}

Bei der Besichtigung einer kleinen Ortskirche (in Gréoux les Bains, Provence) bleibt
Anton ${ }^{1}$ bei seinem Gang durch den Kirchenraum vor zwei großen Gemälden stehen. Die Gemälde hängen raumfüllend in den Bogensegmenten der linken Kirchenwand [Bild 1].

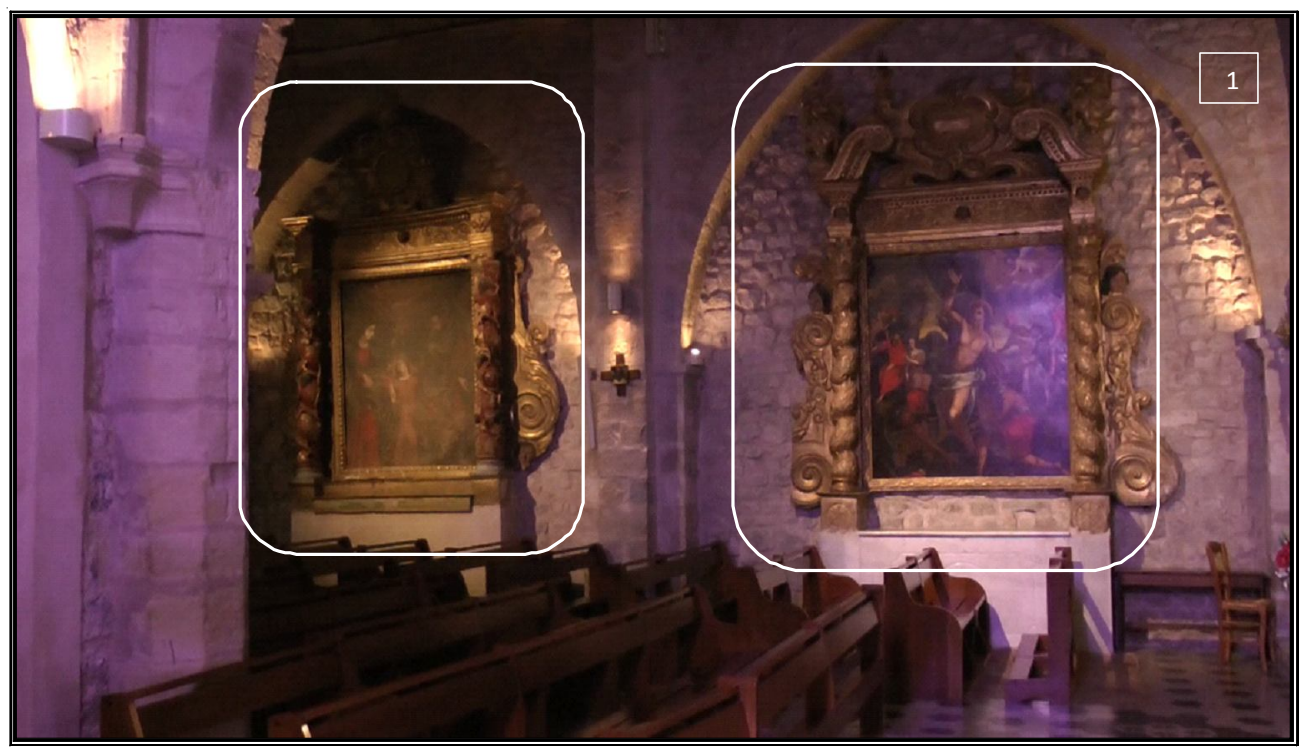


Anton macht die Gemälde für eine gewisse Zeit zum Fokus seiner visuellen Wahrnehmung und thematisiert sie mit wahrnehmungsbegleitenden Kommentaren. Hierdurch konstituiert er sie als relevante Objekte des Kirchenraums und als wesentliche Bestandteile seiner Kirchenbesichtigung. Wir beschäftigen uns ausgehend von dieser einfachen Beobachtung in diesemAufsatz mit folgenden Fragen:

- In welchem räumlichen und wahrnehmungsspezifischen Zusammenhang steht diese Bildbetrachtung?

- In welchen Äußerungsformaten des exothetischen Sprechens kommen die Gemälde zur Sprache? Sprache?

- Welche Aspekte der Bilder kommen zur

- Welche Motivierung lässt sich für die Spezifik finden, in denen die Bilder zur Sprache gebracht werden?

Die empirische Basis, auf der wir diese Fragen beantworten wollen, stammt aus dem Korpus „Kirchenbesichtigungen“, das im Februar und März 2015 in der Provence konstituiert wurde. Unterschiedliche Personen besuchten in der Rolle von Touristen verschiedene Kirchen und kommentierten bei der Besichtigung des Kirchenraums ihre Wahrnehmung. Dabei dokumentierten sie mit einer Actionkamera ihre eigene visuelle Wahrnehmung und wurden gleichzeitig von einer Kontextkamera gefilmt. Das hier zugrundeliegende Material stammt von den Besichtigungen der Dorfkirche in Gréoux.

\section{Kirchenbesichtigung als kulturelle Praktik}

Im theoretischen Rahmen einer Vorstellung von „Kirchenbesichtigung als kulturelle Praktik“ soll (auf der Grundlage einer Analyse des individuellen Umgangs mit den Gemälden) der Aspekt „Objektkonstitution“ als ein zentraler Baustein dieser kulturellen Praktik exemplifiziert werden. Wenn wir von Kirchenbesichtigung als kultureller Praxis sprechen, tangieren wir eine konzeptionelle Vorstellung, die für unterschiedliche Ansätze der Analyse sozialer Interaktion - sei es als Gesprächs-, Diskurs- oder Konversationsanalyse - eine Rolle spielt. Der Kerngedanke dieser Vorstellung besteht in der Konstruktion eines theoretischen Rahmens für die Konzeptualisierung einzelfallanalytischer Ergebnisse als allgemeine, fallunabhängige
Verfahren (zuletzt [Deppermann, Feilke, Linke, 2016]). Diese Grundidee existiert in vielfältiger konzeptioneller Ausarbeitung. Sie wurde für unterschiedlich komplexe interaktive Situationen und Phänomene entwickelt und mit einer Vielzahl von Benennungen versehen. Begrifflich existiert sie beispielsweise als ${ }^{2}$ :

- kommunikative Gattung [Bergmann, Luckmann, 1995; Günthner, 1995, 2006; Günthner, Knoblauch, 1994; Luckmann, 1986, 1988],

-Aktivitätstyp [Levinson, 1979, 1992],

- Handlungsmuster [Ehlich, Rehbein, 1972, 1979],

- Handlungs- und Kommunikationsschema [Heidtmann, 2009; Kallmeyer, 1985, 2000; Kallmeyer, Schütze, 1976, 1977],

- kommunikative und gesprächsrhetorische Verfahren [Schmitt, 2003],

- kommunikative Praktik(en) [Eigenschaften gesprochener Sprache, 2004; Pache, 2004] oder - Sprachhandlungsmuster [Klemm, 2001].

Als generische Bezeichnung dieser unterschiedlichen Verfahren hat sich im sprachwissenschaftlichen Forschungszusammenhang inzwischen „kommunikative Praktik” etabliert. Kommunikative Praktiken werden entweder als Sedimente des interaktiven und gesellschaftlichen Wissens betrachtet (dies gilt insbesondere für die sprachsoziologische Konzeption der kommunikativen Gattung in ihrer Luckmannschen Ursprungsversion) oder sie werden als Bestandteile der Alltagsmethodologie von Interaktionsbeteiligten formuliert (mehr oder weniger klar bezogen auf die konversationsanalytische Konzeption und ihren ethnomethodologischen Ursprung bei Garfinkel [Garfinkel, 1967]).

Obwohl in konzeptioneller und gegenstandkonstituierender Hinsicht unterschiedlich, haben all diese Konzeptionen zumindest zwei Dinge gemeinsam. Sie sind:

- in ihrer Konzeption monomodal und

- in ihrem zentralen Interaktionsbezug handlungsstrukturell.

Monomodal sind sie, weil sie ausschließlich Verbalität als Konstitutionsressource berücksichtigen und nur Formen sozialen Handelns erfassen, die verbal konstituiert sind. Handlungsstrukturell sind sie, weil sie auf die sequenziell geordnete Rekonstruktion von Handlungsschritten fokussiert sind, die als strukturelle Züge oder Konstituenten den übergeordneten Zusammenhang des interaktiv- 
gesellschaftlichen Wissens oder aber die Alltagsmethodologie der Beteiligten repräsentieren.

Vom Standpunkt der multimodalen Interaktionsanalyse aus müssen die Verfahren oder Praktiken jedoch gemäß ihrer faktischen multimodalen Konstitution rekonstruiert und deren Ergebnisse auch multimodal konzeptualisiert werden.

Auf der Grundlage einer solchen multimodalinteraktionsanalytischen Position verstehen wir unter „Kirchenbesichtigung als kulturelle Praktik“ ein komplexes, multimodal-räumliches Unternehmen. Eine Kirchenbesichtigung stellt wie andere kulturelle Praktiken auch - eine situative, handlungsmäßig realisierte, kulturell spezifische und mehr oder weniger explizit vermittelte Wissensstruktur dar. Diese Wissensstruktur bezieht sich - in unserem Fall auf den adäquaten, das heißt sozial verträglichen Vollzug der systematischen Sekundärnutzung sakraler Funktionsräume, die im Normalfall dem Vollzug von Gottesdienst dienen.

Diese kulturelle Praktik kann von Individuen, Paaren oder Kleingruppen realisiert werden. Sie besteht nicht aus einer Führung (und der damit verbundenen Präselektion auf unterschiedlichen Ebenen), sondern wird eigenstrukturiert und auf der Grundlage mitgebrachter Relevanzen realisiert.

Für sie ist eine spezifische Form gezielter visueller Wahrnehmung konstitutiv, die sich in der Regel auf den Innenraum einer Kirche (und nicht wie bei einer Ausstellung auf speziell präsentierte Exponate im Raum) bezieht.

Die Kirchenbesichtigung basiert auf einer Art Besichtigungs-Skript, in dem bestimmte Kernelemente als Objekte gezielter Wahrnehmung in vororientierter Weise enthalten sind (Raumeindruck, Altar, Mittel- und Seitenschiffe,...). Diese Kernelemente werden situativ-dynamisch und bezogen auf die faktische Angebotsstruktur des konkreten Kirchenraums adaptiert und strukturieren so den konkreten Ablauf der visuellen Wahrnehmung sowie die Selektion relevanter Objekte und deren Verbalisierung.

Die Kirchenbesichtigung als kulturelle Praktik besitzt in der Regel eine Struktur, für die das Oszillieren der visuellen Wahrnehmung zwischen dem räumlichen Gesamteindruck und der Detailwahrnehmung einzelner Objekte charakteristisch ist.
Für die kulturelle Praktik sind darüber hinaus die Bewegung im Kirchenraum sowie die stabile Positionierung an bestimmten Orten und das zeitweilige Verweilen vor bestimmten Objekten konstitutiv.

Sie ist zudem mit einer spezifischen, körperlich-interaktiven Präsenz verbunden (generelle Zurückhaltung: keine Mützen, keine Speisen, keine Musik, keine Hunde, leises Sprechen, nicht rennen oder hüpfen, keine Spiele und keine Selfies etc.).

Sie ist Ausdruck einer präsenzstrukturierenden Orientierung an der gottesdienstlichen Primärfunktion des Kirchenraums, bei der die Nutzung der objektiv gegebenen räumlichen Gelegenheiten (im Sinne des Konzepts „Interaktionsarchitektur" und der damit verbundenen Basiskonzepte wie „Begehbarkeit“, „Verweilbarkeit“, „Sichtbarkeit“, „Berührbarkeit“ [Hausendorf, Schmitt, 2013, 2016]) deutlich durch eine sozialtopographische Orientierung bestimmt wird, die in der religiösen Primärnutzung des Raums gründet. Interaktionsarchitektur ist der konzeptionelle Rahmen, in dem rekonstruiert wird, welche Angebote Räume für Interaktion anbieten und nahelegen. Die Frage wird interaktionsvorgängig beantwortet, indem analysiert wird, in welchen Bereichen der Raum überhaupt begehbar ist, wo man sich setzen und verweilen kann, welche Dinge von bestimmten Positionen aus im Raum sichtbar sind bzw. gesehen werden sollen etc.

Sozialtopographie ist hingegen der konzeptionelle Rahmen, in dem konkrete und dokumentierte Formen der Raumnutzung analysiert werden. Diese Nutzungsformen werden hinsichtlich der handlungspraktisch ausgedrückten Wissensgrundlagen der Raumnutzer befragt und theoretisiert. Während die Interaktionsarchitektur große Teile des Altarraums als begehbar ausweist, zeigt die Analyse von faktisch realisierten Laufwegen, dass dieser prinzipiell begehbare Bereich sozialtopographisch, das heißt in Bezug auf sozial relevante Einschränkungen, deutlich strukturiert ist. Das sozialtopographische Wissen, das beispielsweise Konfirmanden bezogen auf die Begehbarkeit des Altarraums der evangelischen Kirche in Rimbach haben, zeigt sich etwa in folgendem Phänomen [Schmitt, 2012a, 2012b]:

Die Konfirmanden folgen der Orientierung, beim Gang zu ihren Kerzen, die sich im rechten Bereich des Altarraums befinden, den vom Altar in den Besucherraum führenden Teppich auf den 
Altarstufen nicht zu betreten. Dies ist keine Frage der objektiven Begehbarkeit des Altarraums, sondern Ausdruck sozialtopographischen Wissens um angemessene Laufwege in diesem Bereich.

Alle dokumentierten Kirchenbesichtigungen weisen eine vergleichbare Dauer auf und unterscheiden sich nur um wenige Minuten. Es scheint also für die ausgewählten Kirchen ein vergleichbares zeitliches Budget zu geben, in dessen Rahmen die kulturelle Praktik realisiert wird. Weder gibt es Schnelldurchgänge, bei denen in unangemessener Präsenzmodalität zu wenig besichtigt wird, noch gibt es zu langes Verweilen, bei dem die Besichtigung in Richtung Exkursion abdriftet. Die Kirchenbesichtigung beginnt im Kern mit dem Eintritt in den Kirchenraum und endet mit dem Verlassen des Kirchen(innen)raums (für die Außenbesichtigung gilt der Aspekt „generelle Zurückhaltung“ nicht mehr). Der Vollzug der kulturellen Praktik setzt ein gewisses Maß an Mobilität, Freizeit, Wahrnehmung als Freizeitaktivität, Kulturkonsum, kirchenraumbezogenes sozialtopographisches Wissen - und natürlich offene Kirchentüren voraus.

\section{Das Erhebungsdesign „Kirchenbesichtigungen“"}

Im Unterschied zur Dokumentation von Interaktionsereignissen, bei denen vor allem die Authentizität der Ereignisse eine zentrale Rolle spielt, sind die Videoaufzeichnungen der Kirchenbesichtigungen unter einer Perspektive entstanden, die sich auf raumlinguistische Grundlagen stützt (siehe [Hausendorf, Schmitt, Kesselheim, 2016] zum aktuellen Stand der Raumlinguistik). Beim Aufbau des Korpus ging es primär darum, adäquate empirische Grundlagen für die Beschäftigung mit der kulturellen Praktik „Kirchenbesichtigung“ zu konstituieren und dabei auch Hinweise auf kognitive Prozesse und die konzeptuellen Grundlagen zu bekommen, welche für die verschiedenen Personen bei der Kirchenbesichtigung eine zentrale Rolle spielen. Vor allem mit den beiden letzten Aspekten war die Entscheidung für ein quasi experimentelles Erhebungsdesign gefallen, bei dem zudem zwei Kameras eingesetzt wurden: eine Actionkamera und eine Kontextkamera.

Die Actionkamera war auf Augenhöhe des Kirchenbesichtigers an einer Kopfseite befestigt.
Sie dokumentierte seinen „point of view“ und zeichnete all das auf, was bei der Kirchenbesichtigung in das Blickfeld der durch den Raum gehenden Person kam und dokumentierte jede noch so kleine Kopfbewegung. Die Kontextkamera folgte dem Träger der Actionkamera bei seinem Gang durch den Kirchenraum. Sie dokumentierte die Person in ihrem spezifischen Gang, ihrer jeweiligen räumlichen Umgebung, ihrer Körperorientierung, ihrem Blickverhalten, ihrer Position und Positur. Beide Kameraperspektiven wurden synchronisiert und stellen somit reichhaltige Informationen über die Kirchenbesichtigung zur Verfügung.

Als Besonderheit der Datenkonstitution wurde wahrnehmungsbegleitendes Sprechen als methodisches Verfahren eingesetzt. Die Kirchenbesichtiger wurden gebeten, während ihres Gangs durch den Kirchenraum ihnen wichtig erscheinende Aspekte ihrer visuellen Wahrnehmung zu verbalisieren. Anders als beim „Selbstgespräch“ oder „Reden mit sich selbst“, das sich in bestimmten Alltagssituationen ungeplant ereignet, handelt es sich hier um ein zielgerichtetes methodisches Verfahren. Von seiner Grundstruktur und seinem Zielbezug hat das exothetische Sprechen gewisse Ähnlichkeiten mit dem „lauten Denken“. Lautes Denken wurde unter anderem in der Sozialpsychologie eingesetzt, um einen Zugang zu kognitiven Verarbeitungsstrukturen (vor allem im Zusammenhang mit Planungs- und Problemlösungsprozessen) von Versuchspersonen zu bekommen (beispielsweise [Konrad, 2010] mit Literaturüberblick). Aber auch in der Translationsund Literaturwissenschaft [Göpferich, 2007; Steen, 1994] sowie der Fremdsprachenforschung [Heine, 2014; Heine, Schramm, 2007; Schreier, 2006] wird mit der Methode des lauten Denkens gearbeitet. Weiterhin gibt es Berührungspunkte mit dem Verfahren der "self-reflective analysis", bei der die Erfahrung des Analytikers selbst (so, wie sie sich in seinen Verbalisierungen zeigt) als ein methodologisches Produkt verstanden und genutzt wird (beispielsweise [Hester, Francis, 2003]).

Die methodische Eigenständigkeit des exothetischen Sprechens besteht vor allem in der Kombination der folgenden Aspekte:

- Es kommt nicht in Laborsituationen zum Einsatz,

- es gibt keine externen Stimuli, um die Exothese am Laufen zu halten, 
- das Verfahren wird nicht in selbstreflexiver Weise eingesetzt, sondern Produzent und Analytiker werden bewusst getrennt.

Die wahrnehmungsbegleitende Verbalisierung erhöht in signifikanter Weise den Nachvollzug der Wahrnehmung des Raumbetrachters. Sie leistet im Grunde genommen die Selektion, Fokussierung und De-Fokussierung bestimmter Aspekte und Ausschnitte aus der Totalität des aktuell im Wahrnehmungsraum des Betrachters Sichtbaren. Die Exothese ersetzt im Medium der Sprache die Arbeit des menschlichen Auges und stellte für die Analyse einen relevanten Rahmen zur Verfügung. Sie rückte einzelne Objekte in den Vordergrund und defokussierte gleichzeitig alles im Moment nicht Relevante, rückte es - analog dem menschlichen Auge - in den Unschärfebereich der Peripherie oder des Hintergrunds. Ohne wahrnehmungsbegleitende Verbalisierung bleibt aufgrund der undifferenzierten Relevanzstruktur sehr Vieles im Vagen, Hypothetischen und Vieldeutigen.

Es sind vor allem die verbalen Thematisierungen, die Hinweise auf die relevanten Orientierungen und kognitiven Aktivitäten der Kirchenbesichtiger liefern und somit eine empirisch evidente Rekonstruktion des der Besichtigung zugrundeliegenden Konzepts ermöglichen. $\mathrm{Zu}$ hören, was Anton beim Blick zu den Kirchenfenstern sagt, welche sprachlichen Formate er dabei benutzt, ob eventuell evaluative Äußerungen als Ausdruck des Stellenwerts der Ästhetik für seine spezifische Realisierung der kulturellen Praktik eine Rolle spielen, ist zusätzlich zur kameratechnischen Dokumentation seines Wahrnehmungsraums eine notwendige Bedingung für den Versuch, sein spezifisches Konzept „Kirchenbesichtigung“ zu rekonstruieren.

\section{Experimentelles Erhebungsdesign und Authentizitätspostulat}

Bei einem Erhebungsdesign, das mit einer expliziten Kommentierungsaufgabe operiert, indem es die Kirchenbesichtiger zur wahrnehmungsbegleitenden Verbalisierung auffordert, stellt sich die folgende Frage: Was kann man mit den produzierten Daten analytisch anfangen? Bei der Beantwortung dieser Frage ist es sinnvoll, sich mit der von der Konversationsanalyse formulierten
Authentizitätsanforderung für ihre empirischen Grundlagen zu beschäftigen.

\subsection{Authentizitätspostulat der Konversationsanalyse.}

Die Konversationsanalyse stellt für die Qualität ihrer empirischen Grundlagen die Forderung, nur mit authentischen Daten zu arbeiten, die in ihrer - von der Dokumentation gänzlich unabhängigen und (im besten Fall auch unbeeinflussten) - natürlichen, alltagsweltlichen Umgebung entstanden sind. $\mathrm{Zu}$ dieser alltagsweltlichen Umgebung zählen räumlichmaterielle, soziale und auch kulturelle Aspekte. Die zentrale Forderung im Rahmen dieses Authentizitätspostulats lautet: Die Daten müssen naturally occurring $\operatorname{sein}^{3}$. Diese Authentizitätsforderung ergibt sich für die Konversationsanalyse aufgrund ihres zentralen Erkenntnisinteresses. Wenn es darum geht, die von den Interaktionsteilnehmern bei der Hervorbringung ihrer sozialen Realität eingesetzten Methoden zu rekonstruieren, dann darf die Realisierung dieser Methoden nicht durch einen „fremdgesetzten“ Impuls beeinflusst werden.

Die zentrale Annahme ist dabei, dass nur alltagsweltliche Relevanzen, Ziele und Zwecke, zu deren Verfolgung die Interaktionsbeteiligten gemeinsam Interaktionskonstitution betreiben, den Vollzug dieser Methoden in ihrer authentischen alltagsweltlichen Qualität, Struktur und Vollzugscharakteristik hervorbringen. Wird hingegen der Vollzug dieser Methoden für die Zwecke der Datenkonstitution initiiert, verfälscht das die Daten und sie verlieren ihre Authentizität. Bei solchen bewusst initiierten Daten analysiert man letztlich ein Artefakt und nicht mehr die tatsächliche Realität. Die klassische Diskussion um das „Beobachterparadoxon" ${ }^{4}$ fasst den „,verfälschenden“ Einfluss der Anwesenheit/ Dokumentation auf das authentische Interaktionsverhalten prägnant zusammen: Das Ziel, authentisches Verhalten zu beobachten und zu dokumentieren, wird durch die Tatsache der Dokumentation verhindert bzw. gefährdet.

4.2. Die erkenntnisgebundene Relevanz des Authentizitätspostulats.

So, wie die Konversationsanalyse speziell für die Untersuchung ihrer Erkenntnisinteressen adäquate Daten konstituiert, konstituieren wir im Rahmen unserer spezifischen Erkenntnisinteressen (hier die Rekonstruktion der den Kirchenbesichtigungen 
zugrundeliegenden handlungsleitenden Orientierungen) ebenfalls adäquate Daten. Authentizität ist also keine generelle Anforderung an die Dokumentation interaktiv-sozialer Praxis allgemein. Sie ist vielmehr die zentrale Voraussetzung für die empirischeAnalyse der basalen Mechanismen der Interaktionskonstitution. Andere Erkenntnisinteressen fordern andere Voraussetzungen und geben andere Antworten auf die empirische Adäquatheitsanforderung, die für jegliche Form der Datenkonstitution gilt. Ein Zugang zu den Besichtigungskonzepten der Kirchenbesucher/innen ist auf der Basis der ausschließlichen Dokumentation ihrer visuellen Wahrnehmung, ihrer Laufwege und Bewegungen im Raum und den dabei eingenommenen Positionen und Posituren nicht möglich. Das ist eine der zentralen Erkenntnisse der systematischen Reflexion des Konstitutionsprozesses beim Kirchenbesichtigungs-Korpus.

Denn hinsichtlich der visuell beobachtbaren und dokumentierbaren Präsenz im Kirchenraum verhalten sich alle Kirchenbesichtiger mehr oder weniger gleich. Zudem kann man das, was sie visuell wahrnehmbar tun, nicht in angemessener Weise - bezogen auf ihre eigenen Besichtigungsrelevanzen - interpretieren ${ }^{5}$. Das ändert sich in dem Moment, in dem man hören kann, was die Kirchenraumbesichtiger wahrnehmungsbegleitend verbalisieren. Dann wird schlagartig deutlich, dass ihre mitgebrachten Relevanzen und die Strukturen ihres BesichtigungsSkripts sehr unterschiedlich sein können.

Es geht also um die Notwendigkeit der Produktion adäquater empirischer Grundlagen für die
Untersuchung spezifischer Fragestellungen, die im konversationsanalytischen Erkenntniszusammenhang in motivierter Weise zum Authentizitätspostulat geführt hat.

Weiterhin wird durch das Erhebungsdesign ein Aspekt berührt, der im konversationsanalytischen Zusammenhang durchaus kontrovers diskutiert wird: das Interesse an sprachlichen Manifestationen als Hinweise auf kognitive Relevanzen. Es dürfte klar geworden sein, dass es hier nicht um die von Deppermann [Deppermann, 2012] formulierte, für das konversationsanalytische Erkenntnisinteresse relevante Frage geht ,How does , cognition ' matter to the analysis of talk-in-interaction?". Wir befinden uns nicht im konversationsanalytischen Erkenntniszusammenhang, sondern stellen bewusst eine Situation her, in der die sprachliche Repräsentation von Kognition im Mittelpunkt des Erkenntnisinteresses steht und die zentrale Voraussetzung dafür darstellt, Zugang zu den Besichtigungskonzepten der Kirchenbesucher zu bekommen.

Die nachfolgende Grafik stellt den Zusammenhang von allgemeiner, empirischer Adäquatheitsanforderung und den Antworten der Konversationsanalyse (Authentizitätsforderung) und Kirchenbesichtigung (quasi experimentelles Erhebungsdesign) dar. Dabei wird deutlich: Die Forderung nach Authentizität ihrer Daten ist die Antwort der Konversationsanalyse auf die übergeordnete - und für alle Formen der Datenkonstitution geltende - empirische Adäquatheitsanforderung relativ zu ihren spezifischen Erkenntnisinteressen.

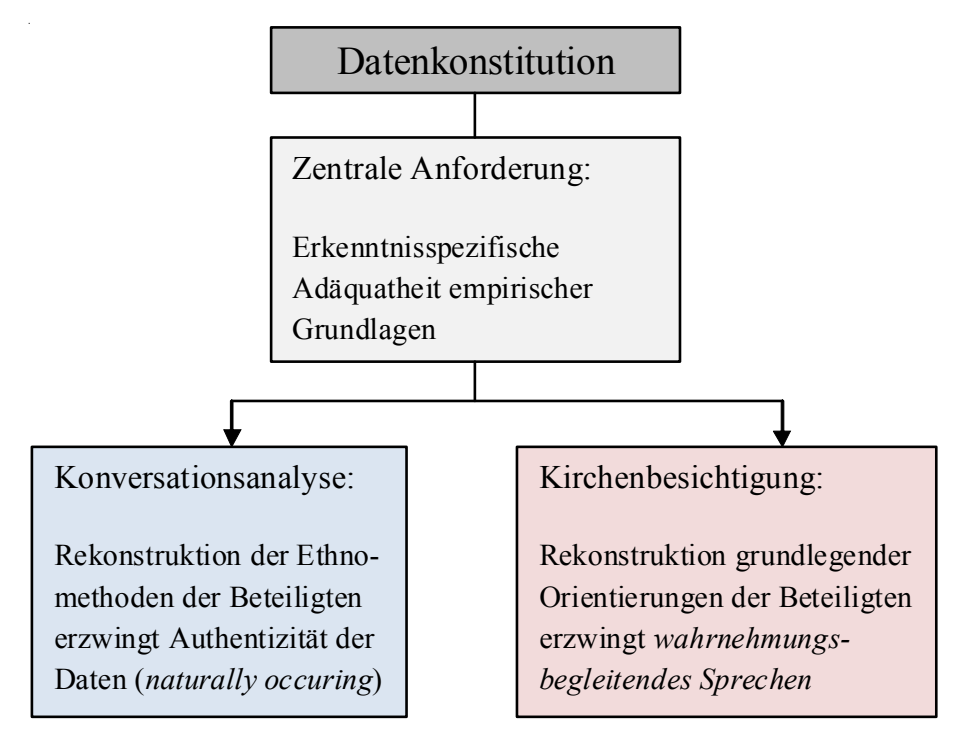




\subsection{Empirische Adäquatheit: Eine erkenntnisunabhängige Anforderung.}

Die Bearbeitung der Anforderung der „erkenntnisspezifischen Adäquatheit empirischer Grundlagen" führte bei der Konstitution des Korpus „Kirchenbesichtigungen“ zu einem quasi experimentellen Design. Dieses entstand als Antwort auf die Adäquatheitsforderung an die empirischen Grundlagen imLaufe der Aufnahmenzu diesemKorpus. Ausschlaggebend hierfür war das Erkenntnisinteresse, Zugang zu den orientierungsleitenden Grundlagen zu bekommen, welche die Besichtigung eines Kirchenraums strukturieren.

Die Kirchenbesichtigung war letztlich motiviert durch die Bearbeitung einer Aufgabe. Die dabei produzierten Daten sind das Ergebnis der Bearbeitung und nur in dieser Qualität aufschlussreich und adäquat. Denn nur über die kontinuierliche, die visuelle Wahrnehmung begleitende und strukturierende Verbalisierung wurde dieser Zugang eröffnet. Es wäre daher sinnfrei, die Daten hinsichtlich ihrer Authentizität zu beurteilen. Denn im hiesigen Erkenntniszusammenhang spielen Aspekte der Interaktionskonstitution, so wie sie in der Konversationsanalyse untersucht werden - und damit auch die Frage nach der für die Konversationsanalyse wichtigen Authentizität der empirischen Grundlagen keine Rolle.

Mit dem Einsatz des exothetischen Sprechens als Erhebungsmethode haben wir den Kirchenbesuchern letztlich die Besichtigung methodisch motiviert erschwert. Wir haben sie dazu gebracht, über die wahrnehmungsbegleitende Verbalisierung zum Ausdruck zu bringen, welche Problemlösungsgrundlagen sie anzapfen und welche Problemlösungsverfahren sie bei der Besichtigung realisieren. Im Hintergrund stand die - im ethnomethodologischen Verständnis motivierte Annahme, dass die Bearbeitung der gestellten Aufgabe dieQualität einer methodischen Hervorbringung besitzt und dass es auch hier so etwas wie „order at all points" als relevante Analyseperspektive gibt.

\section{Die Fallanalyse}

Wenden wir uns nun der angekündigten Analyse der „Bildbetrachtung” von Anton zu ${ }^{6}$.

\subsection{Konstitution der Gemälde als} „Bilderrahmen".

Wir haben eingangs darauf verwiesen, dass wir keine isolierten Bildbetrachtungen analysieren, sondern den Umgang mit den Gemälden als Objektkonstitution rekonstruieren wollen. Deshalb gehört zur Analyse auch die Berücksichtigung des bereits zurückgelegten Laufwegs, die Positionierungen vor den beiden Gemälden sowie die Abwendung von den Bildern und die Fortsetzung des Laufwegs. Und angesichts der Relevanz, welche situationseröffnende Sequenzen als ,mikrostrukturelle Verdichtungen“ relevanter Aspekte des Gesamtdokumentes besitzen, wollen wir zunächst einen Blick auf die ersten exothetischen Aktivitäten Antons richten.

Wenn wir in diesem Zusammenhang von Objektkonstitution sprechen, dann meinen wir damit Folgendes: Die Gemälde werden von ihrem Betrachter nicht einfach als Aspekte der räumlichen Umgebung wahrgenommen, sondern durch die Art ihrer visuellen und verbalen Fokussierung als spezifische Objekte relevant gesetzt. Diese Objektkonstitution läuft primär über die Dynamik von Objektselektion und Objektfokussierung und erschafft diese - in einem gewissen Rahmen - relativ zu den mitgebrachten Besichtigungsrelevanzen neu.

Wir beginnen nun die Analyse mit der Verortung der bei der Bildbetrachtung eingenommenen Positionen im Kontext des bereits von Anton zurückgelegten Laufwegs.

\subsubsection{Situationseröffnung, Positionen} und Laufwege.

Das nachfolgende Verbaltranskript ${ }^{7}$ zeigt Antons thematische Situationseröffnung.

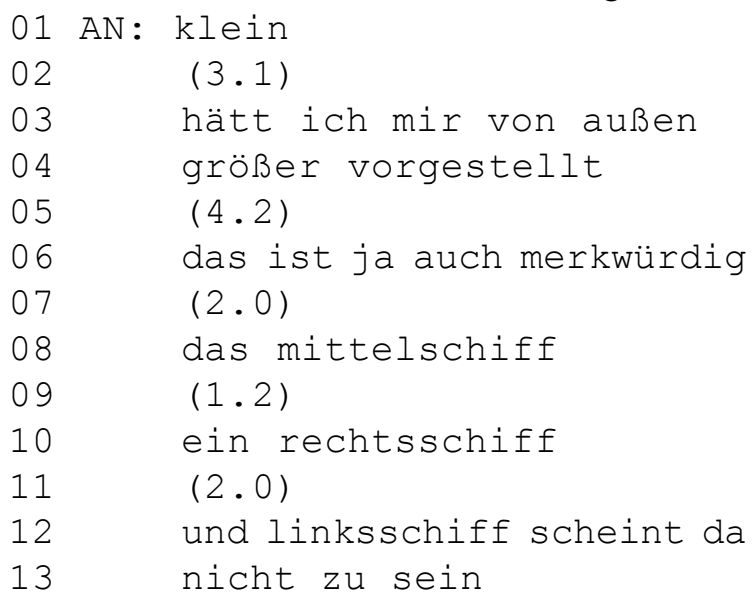

Wir sehen, dass er unmittelbar mit einer Thematisierung und Evaluation des Gesamtraums einsteigt, den er zunächst mit klein formuliert. Für Anton spielt also die Größe des kirchlichen Innenraums als eine Primärorientierung eine wichtige Rolle. Er beendet anschließend die 
Sprechpause mit der Äußerung hätt ich mir von außen größer vorgestellt. Damit macht er nicht nur klar, dass er bestimmte Erwartungen an den Innenraum (und seine Größe) mitgebracht hat, sondern, auch, dass diese Erwartungen eine Folge der Außensicht des Kirchengebäudes sind. Diese Erwartungen werden nun enttäuscht.

Und es folgt sogleich ein weiterer Erwartungsbruch: In Antons Vorstellung existiert die Erwartung, dass eine Kirche mit einem rechten Seitenschiff auch ein linkes Seitenschiff besitzt. Dies ist jedoch nicht nur ganz offensichtlich nicht der Fall, sondern auch merkwürdig. Er zählt dann die vorhandenen Schiffe als basale Einheiten des architektonischen Gesamtraums auf: das mittelschiff, ein rechtsschiff. Anschließend konstatiert er mit und linksschiff scheint da nicht zu sein eine relevante Abwesenheit relativ zu seiner Erwartung.

Betrachtet man den weiteren Fortgang seiner exothetischen Aktivitäten, dann zeigt sich, dass Anton auch weiterhin mit der Bearbeitung seiner nicht erfüllten Erwartungen beschäftigt ist. Er hat während seiner verbalen Situationseröffnung nicht nur die Kirche betreten und ist die Treppe hinabgestiegen, sondern hat sich am Fuß der Treppe im Eingangsbereich positioniert. Hier bleibt er eine Weile stehen und verschafft sich einen Überblick über den Kircheninnenraum (Position 1). Dann geht er langsam und schlendernd im Mittelgang nach vorne. Zum zweiten Mal kommt er bei der Orgel zum Stand und verweilt hier einige Zeit (Position 2). Er setzt sich dann wieder in Bewegung, bleibt jedoch gleich wieder stehen und positioniert sich vor der Säule rechts neben der Orgel (Position 3).

Er betrachtet das kleine Holzkreuz, das hier wie an allen anderen Säulen - angebracht ist [Bild $2]^{8}$. Er macht dann einige Schritte im Mittelgang nach vorne und bleibt bei einem schmalen Quergang stehen (Position 4), von wo aus er feststellt, dass der Raum auf der linken Seite kein Seitenschiff aufweist.

14 AN: und hier (.)

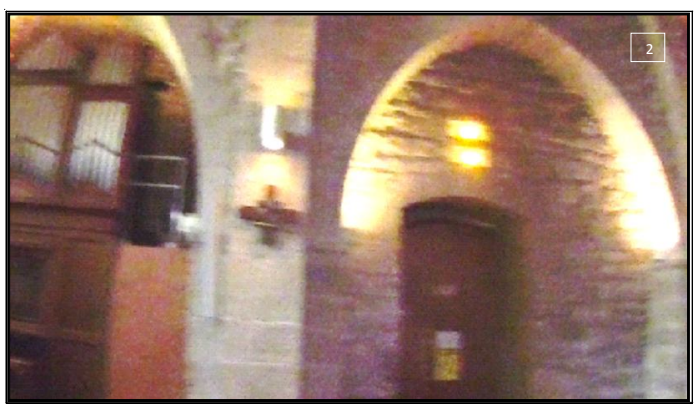

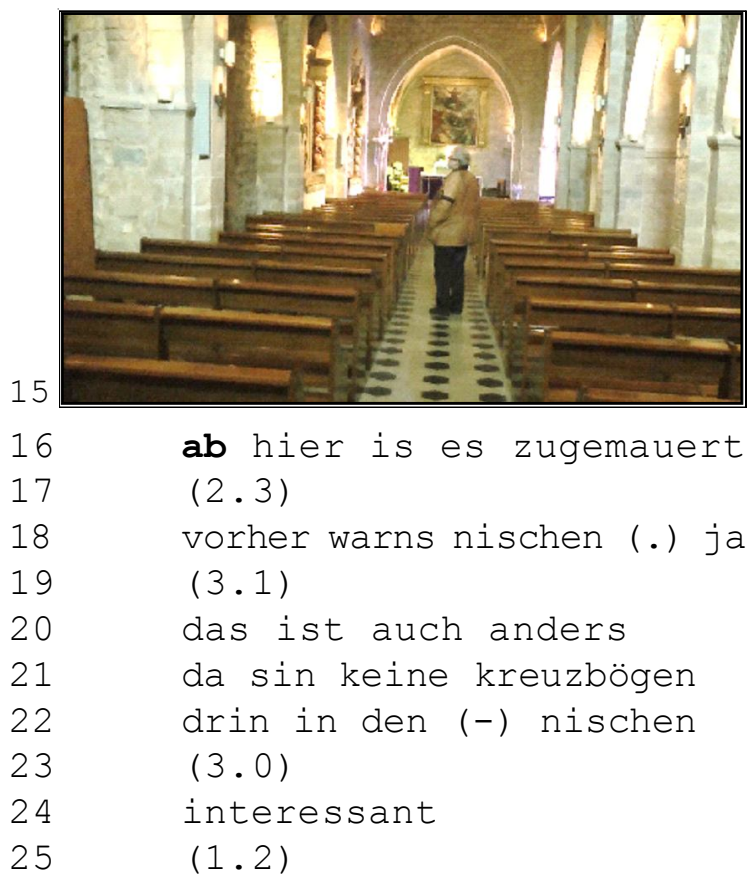

Ein interessanter Aspekt, der die Stärke seiner Erwartungen zeigt, die er hinsichtlich der symmetrischen und harmonischen Gestaltung der Grundstruktur des Kirchenraums hat, ist eine explizite Normalisierung seiner Wahrnehmung. Mit $a b$ hier ist es zugemauert formuliert er selbst eine Erklärung für das fehlende Linksschiff. Durch die Spezifik seiner Formulierung ab hier ist es (das Linksschiff, R. S.) zugemauert kann Anton seine Erwartung „retten“ und das normative Modell „seines" Kirchenraums aufrechterhalten: Das Linksschiff war - wie in seiner Erwartung vorgesehen - einmal vorhanden und wurde im Zuge eines Umbaus zugemauert.

Anton ist aufgrund seiner Suche nach einem linken Seitenschiff auf die linke Raumseite orientiert und blickt deshalb beim Vorwärtsgehen in den linken Wandbereich, in dem die beiden Gemälde hängen. Als er schließlich bei dem ersten Gemälde zum Stand kommt, ist das die fünfte Position auf seinem Gang durch den Kirchenraum (Position 5).

5.1.2. Visuelle Fokussierung und verbale Thematisierung.

Das nachfolgende multimediale Transkript ${ }^{9}$ (Zeile 26-63) zeigt Antons Annäherung an die beiden Gemälde. Einige Zeit vor seiner ersten Thematisierung hat Anton die Gemälde bereits in den Blick genommen. 
26 $(8.0)$
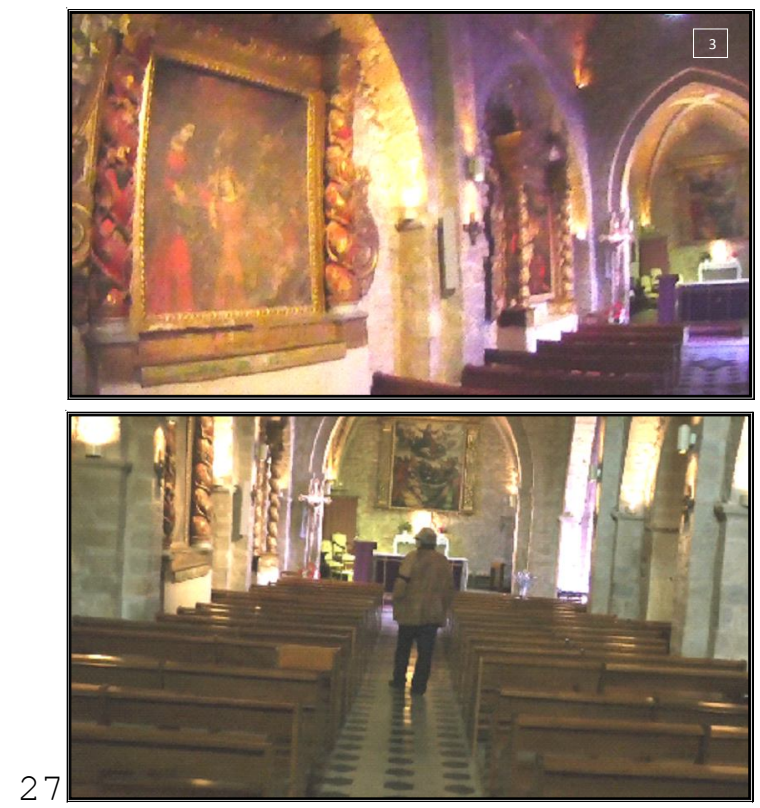

28 AN: riesige bilder

Seine erste Äußerung erfolgt noch in der Phase der Annäherung. Die beiden Standbilder [Bild 3] zeigen die allmähliche visuelle Fokussierung der beiden Gemälde. Zu diesem Zeitpunkt ist aufgrund seiner Körperorientierung, die stärker nach rechts-vorne ausgerichtet ist und auch aufgrund seiner Bein- und Fußstellung nicht klar, ob er sich vor dem Gemälde verankern oder seinen Weg nach vorne in Richtung Altarraum fortsetzen wird.

Anton thematisiert die beiden Gemälde also, bevor er eine stabile Position eingenommen hat. Er weist die Größe der Gemälde (riesige bilder) als deren erstes, bestimmendes Merkmal aus. Dass er gleich beide Gemälde thematisiert, ist Ausdruck dafür, dass sie für ihn aufgrund ihrer Objektqualität zusammengehören. Was die thematische Relevanz der Größe der Gemälde betrifft, so hängt sie nur bedingt mit ihren objektiven Ausmaßen zusammen. Vielmehr spielt neben ihrem Flächenverhältnis zur Wand, an der sie befestigt sind, letztlich die gesamte Proportionalität der Raumstruktur eine Rolle. Dies wird beispielsweise daran deutlich, dass die wesentlich größeren Bilder im linken Seitenschiff der Kirche in Manosque keine vergleichbare Thematisierung erfahren. Hier scheint das breite und hohe Seitenschiff ihre Größe zu relativieren und eine Thematisierung zu verhindern ${ }^{10}$.

Die thematische Relevanz der Gemälde erfolgt als aktive Objektkonstitution im Kontext der Besichtigung dieses spezifischen Kirchenraums. Die Größe der Gemälde (nicht etwa „groß“, sondern ,riesig“) verweist darauf, dass sie nicht einfach als eigenwertige Objekte mit bestimmten Eigenschaften relevant gesetzt, sondern im Kontext ihres unmittelbaren und weiteren architektonischen Zusammenhangs mit dieser spezifischen Qualität ausgestattet und entsprechend bewertet werden. Dies geht gut einher mit Antons Äußerung hätte ich mir von außen größer vorgestellt, die er im Kontext seiner Erstpositionierung am Fuße der Treppe realisiert. Die Äußerung thematisiert als ersten Aspekt des Kirchenraums überhaupt die Tatsache, dass sie recht klein ist. In dieser räumlichen Kleinheit erscheinen dann wandsegmentgroße Gemälde als riesig.

Die gleichzeitige Thematisierung beider Gemälde ist zum einen Ausdruck einer objektkategorialen Sicht. Unter dieser Perspektive gehören Gemälde, die an einer Wand hängen, eher zusammen als andere Objekte, die ebenfalls an der Wand angebracht sind. Dass sie gleich zusammen in den Blick kommen und dann auch in die Sprache gelangen, hat zum anderen auch mit Antons segmentaler Orientierung auf den Kircheninnenraum zu tun. Nur wenn Anton bei einer visuellen Segmentierung des Raums eine Einheit - wie etwa die linke Wand - als relevanten Wahrnehmungsraum konstituiert, fallen beide Gemälde als markante binnenstrukturelle Elemente dieses Segments gemeinsam und gleichzeitig auf - und können thematisiert werden.

Für Antons segmentale Sicht spielt - das hat der bereits zitierte Transkriptausschnitt mit der „Erklärung“ für das fehlende Linksschiff ( $a b$ hier ist es (das Linksschiff, R.S.) zugemauert; Zeile 16) verdeutlicht - eine auf architektonische Strukturen ausgerichtete Wahrnehmung eine wesentliche Rolle. Dass dabei die linke Wand als wichtiges Segment des Raums - und damit auch die Gemälde - in den Blick kommen, ist eben auch durch die Suche nach dem (nicht vorhandenen) zweiten Seitenschiff motiviert.

Als einen relevanten Punkt wollen wir an dieser Stelle festhalten, dass die gleichzeitige Thematisierung beider Gemälde Ausdruck einer raumsegmentierenden Wahrnehmung ist, die auf Antons architekturbasiertem Konzept beruht, über das er sich in einem ersten Angang den Kirchenraum strukturell erschließt. Erst in einem 
zweiten Schritt geht Anton zu einer objektorientierten Wahrnehmung und Thematisierung über.

In der nachfolgenden Sprechpause (3.0) wandert sein Blick nach rechts zum zweiten Gemälde, wobei große Teile des Altarraums Bestandteil seines visuellen Wahrnehmungsraums sind [Bild 4]. Hätte man nur dieses Standbild zur Verfügung, würde man den Eindruck gewinnen, Antons Blick streife das zweite Gemälde nur auf seinem Weg nach vorne. Aufgrund der linksseitigen Fokussierung des Gemäldes durch die Actionkamera vermutet man hier eher eine Vororientierung auf den Altarraum als eine längere Beschäftigung mit den Gemälden.
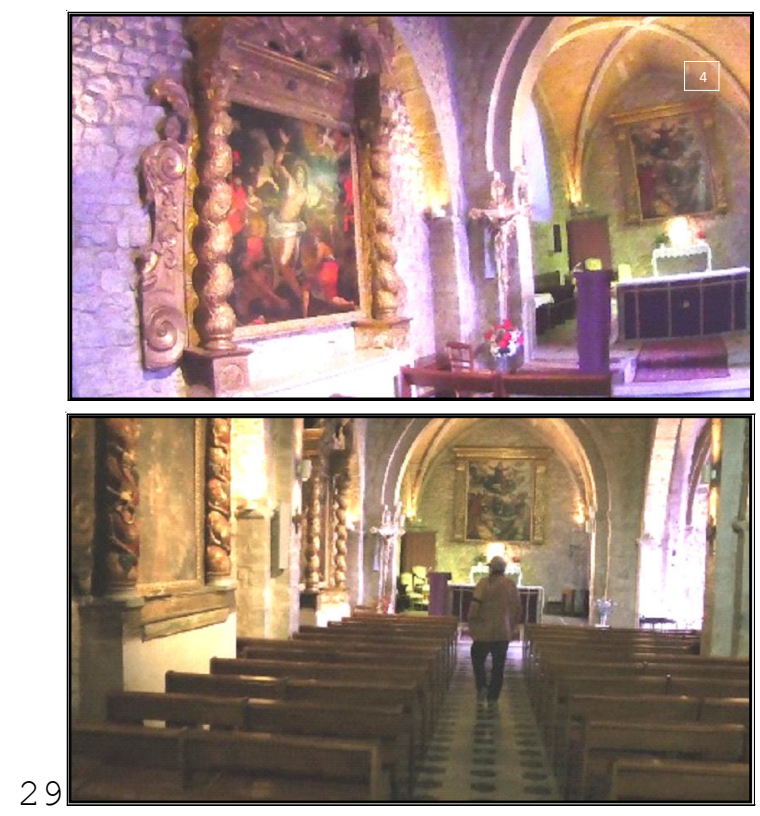

30

(3.0)

Anton bleibt jedoch bei der Beschäftigung mit den Gemälden und orientiert sich wieder zurück auf das erste Bild. Mit dieser Rückorientierung erfolgt nun auch der Übergang von einer segmentalen Raumorientierung zu einer auf einzelne Objekte fokussierten Wahrnehmung. Diese Veränderung ist in seiner visuellen Wahrnehmung deutlicher sichtbar als im verbalen Ausdruck. Anton hat sich jetzt vor dem ersten Bild verankert und seinen Körper auf das Gemälde ausgerichtet [Bild 5]. Die visuelle und verbale Konzentration auf das erste Bild geht also einher mit der Veränderung seiner Präsenzform: Er ist nicht mehr in Bewegung, sondern steht nun auf die linke Wand der Kirche ausgerichtet mit beiden Füßen in Parallelstellung im Mittelgang.
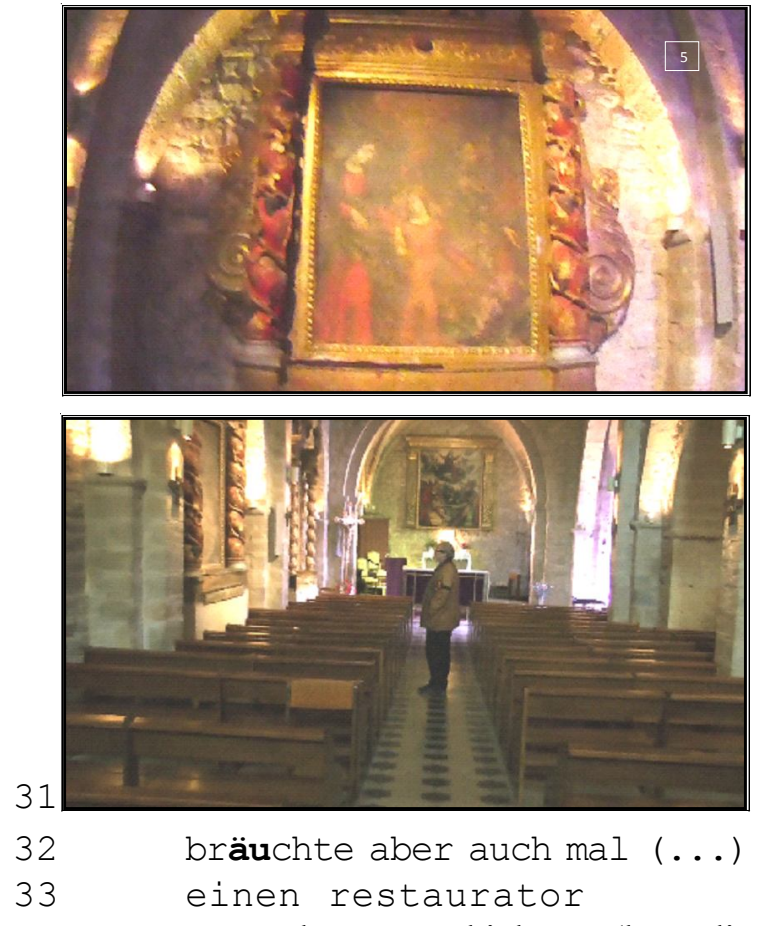

Man muss schon genau hinhören (bzw. die Äußerung im Transkript genau lesen), um die Bewegung von den beiden Gemälden zum ersten Gemälde zu erkennen. Die Äußerungsstruktur legt nämlich - vor allem durch das aber - die Lesart nahe, die begonnene Äußerung würde sich als Teil einer Fokusopposition auf die beiden Gemälde gleichermaßen beziehen. Antons ausschließliche visuelle Fokussierung des linken Gemäldes im Dokument der Actionkamera zeigt jedoch, dass dies nicht der Fall ist. Es ist also die visuelle Wahrnehmung, die gegenüber der verbalen Thematisierung den faktischen Fokuswechsel verdeutlicht. Anton gibt nun seinen segmentalen Blick auf den Raum und die Gemälde und deren Pluralthematisierung auf und beschäftigt sich nur noch mit einem (dem ersten) Gemälde: Nicht beide, sondern nur das eine Gemälde bräuchte aber auch mal (...) einen restaurator.

$34(1.3)$

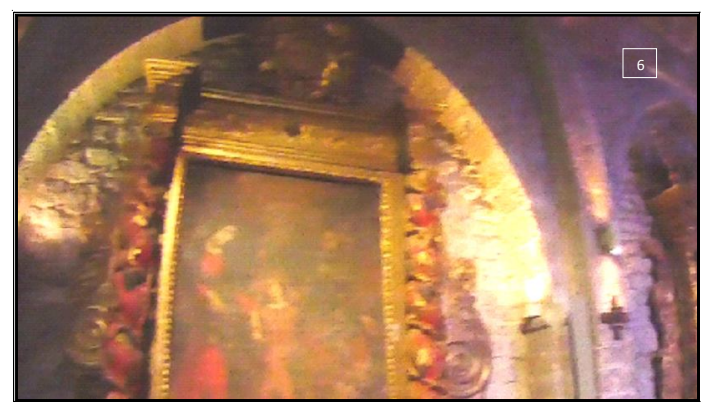




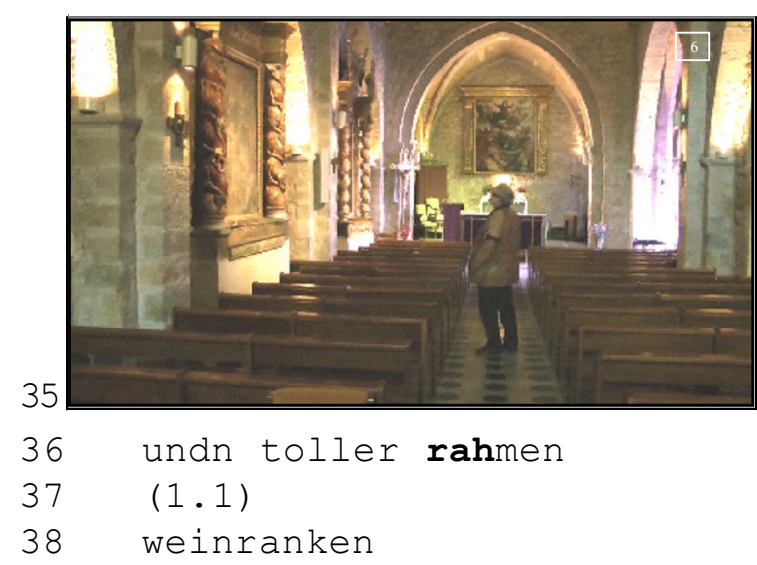

Antons Fokussierung auf das Gemälde wird nun spezifischer und detaillierter. Nach der Thematisierung des Zustandes des Gesamtgemäldes beschäftigt er sich anschließend nur noch mit dem Rahmen undn toller rahmen. Im Standbild bildet sich die zunehmende Fokussierung der visuellen Wahrnehmung in einem leichten Schwenk der Actionkamera nach oben ab [Bild 6]. Hatte man im Standbild zuvor noch das ganze Gemälde gesehen, so ist es nun unten etwas abgeschnitten. Im Dokument der Kontextkamera ist deutlich zu sehen, wie Anton seinen Kopf in den Nacken legt.

Das und in Erstposition der Äußerung suggeriert, Anton habe bereits etwas über das erste Bild ausgesagt und füge nun mit dem rahmen den nächsten Aspekt an. Das ist zwar zutreffend, denn Anton hat bereits die Restaurationsbedürftigkeit des Gemäldes verbalisiert. So ganz will der Anschluss mit und dennoch nicht passen, denn mit der und-Äußerung folgt eine Positivevaluation auf eine Negativevaluation. Eine solche Beziehung hätte also in einem aber einen adäquateren Anschluss.

Dieser auf den ersten Blick nicht ganz passende Anschluss eröffnet einen Einblick in einen interessanten Zusammenhang, der das Verhältnis von visueller Wahrnehmung und verbaler Thematisierung betrifft. Will man nicht einfach über die Anschlussimplikationen des erstpositionalen und hinweggehen und zieht gleichzeitig in Betracht, dass nicht alles visuell Wahrgenommene auch thematisiert wird, ergibt sich folgende Lesart: Das und indiziert einen Anschluss, dessen Bezugsaspekt nicht im Bereich verbaler Vorgängigkeit, sondern im Bereich visueller Wahrnehmung liegt. Verbale Thematisierungen sind im Rahmen der Kirchenbesichtigung und im Kontext der mit der Besichtigung assoziierten Aufgabe zumindest doppelt anschließbar. Es besteht die Möglichkeit, äußerungseröffnende Anschlüsse zu produzieren, die keine „passenden“ verbalen, sondern visuelle Bezugspunkte besitzen. Dies ist ein wichtiger und grundlegender Aspekt des Verhältnisses von visueller Wahrnehmung, kognitiver Aktivität und verbaler Thematisierung.

Die visuelle Wahrnehmung muss aus der Totalität des Wahrnehmbaren auswählen und einzelne Objekte, Aspekte oder Zusammenhänge fokussieren (und dabei automatisch andere abwählen). Gleiches gilt auch für das auf die visuelle Wahrnehmung bezogene exothetische Sprechen. Auch bei der verbalen Thematisierung müssen bestimmte Objekte, Aspekte oder Zusammenhänge für die Thematisierung ausgewählt (und andere damit abgewählt) werden. Diese aspektuelle Thematisierung bedeutet nun jedoch nicht, dass die bei der Exothese abgewählten (jedoch visuell wahrgenommenen) Aspekte gänzlich unwichtig oder unwirksam wären. Sie besitzen - nur eben in einem anderen Medium - durchaus die Relevanz wichtiger Bezugspunkte. Würde alles visuell Wahrgenommene tatsächlich auch verbal thematisiert, wären die Kirchenbesichtigungen nicht nur wesentlich länger, sondern die Kirchenbesichtiger würden sich kontinuierlich am Rande der kognitiven Überlastung befinden.

Doch nicht nur selektive Gleich- und Nahzeitigkeit ist ein wichtiger Aspekt des Verhältnisses von visueller Wahrnehmung und verbaler Thematisierung. Hierzu gehört weiterhin auch lokale Bi-Fokalität, bei der visuelle Wahrnehmung und verbale Thematisierung zwei gänzlich eigenständige Foki besitzen. Ein instruktives Beispiel hierfür liefert Anton einige Zeit später. Während er zunächst das im Altarraum stehende Vorlesepult betrachtet und dann für längere Zeit ein im Altaraußenbereich stehendes Kruzifix ins Auge fasst, produziert er die Äußerung keine kanzel. Es ist offensichtlich, dass sich die Äußerung nicht auf das simultan wahrgenommene Kruzifix bezieht, sondern die Tatsache thematisiert, dass die Dorfkirche nicht über eine Kanzel verfügt. Da das Kruzifix nicht an einer für eine Kanzel systematischen Stelle steht, kann auch seine Position kein Motiv für die Thematisierung sein. Begreift man jedoch das Vorlesepult als einen systematischen und interaktionsarchitektonisch ausgewiesenen 
Sprechort, dann kann das Pult die fehlende Kanzel - einen weiteren zentralen Sprechort (für die Predigt) - motivieren.

Visuelle Wahrnehmung und verbale Thematisierung können also temporär eigenständige Foki haben. Man sollte sich daher bei der Analyse und Konzeptualisierung des Zusammenhangs davor hüten, von einer fraglosen Gleichzeitigkeit von visueller Wahrnehmung und wahrnehmungsbegleitender Thematisierung auszugehen.

In der Einwortäußerung weinranken, die in ihrem kondensierten und aspektualisierten Format dem Ausmaß der visuellen Fokussierung entspricht, thematisiert Anton anschließend ein Detail des Rahmens. Wir sehen hier eine enge Synchronisierung von visueller Wahrnehmung und verbaler Thematisierung. Dabei bildet sich in der Exothese die zunehmende Enge des visuellen Fokus in dem stark reduzierten verbalen Format $a b$. Insgesamt besteht die Dynamik dieser sukzessiven Detaillierung aus folgenden Schritten:

1. die beiden Gemälde,

2. das erste Gemälde,

3. der Rahmen des ersten Gemäldes und

4. die Weinranken als ein Aspekt des Rahmens des ersten Gemäldes.

Anton schließt dann - im Weitergehen - die Beschäftigung mit dem Gemälde mit der Positivevaluation intressant ab, wobei er auch hier wieder auf das sprachliche Format der Einwortäußerung zurückgreift. Es bleibt im Unbestimmten, worauf sich seine positive Bewertung bezieht. Berücksichtigt man die Aspekte seiner verbalen Thematisierung, dann kommen folgende Kandidaten in Betracht: die Größe des Gemäldes, der Rahmen oder die Weinranken. Es ist jedoch nicht zwingend, sich ausschließlich auf Antons Exothese zu beziehen, denn es kann auch ein Aspekt oder ein Zusammenhang sein, der nicht thematisiert, aber wahrgenommen wurde.

Die Konsistenz- und Kohärenzanforderungen an die verbale Thematisierung des visuell Wahrgenommenen scheinen für Anton nicht primär $\mathrm{zu}$ sein. Wenn man daraus eine erste Arbeitshypothese ableiten wollte, könnte man sagen: In der Spezifik, in der die Notwendigkeit, die visuelle Wahrnehmung begleitend zu kommentieren, bearbeitet wird, dokumentiert sich eine Selektivität, für die primär mitgebrachte
Relevanzen verantwortlich sind. Diese relevanzbasierte Selektivität ist nicht nur dafür verantwortlich, welche wahrgenommenen Aspekte in welchem Format thematisiert werden. Sie bestimmt auch, an welche Aspekte Formulierungen jeweils angeschlossen werden. Man muss dabei grundsätzlich von mehr Anschlussmöglichkeiten ausgehen als die Äußerungsstruktur selbst anbietet.
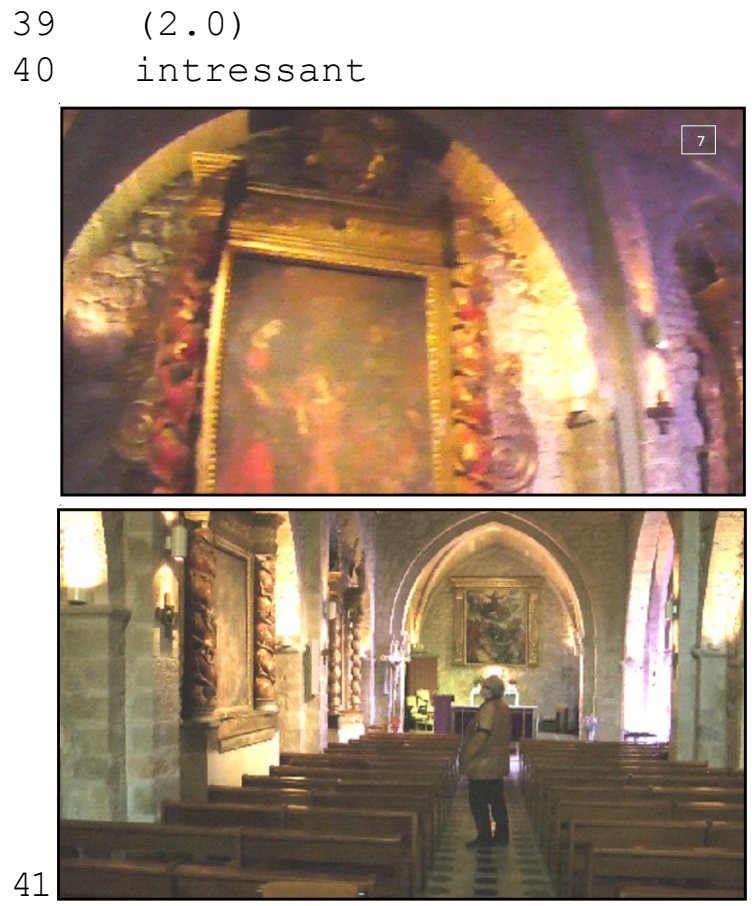

42 $(1.0)$

$43 \quad(5.4)$

Bis zum Beginn der Sprechpause nach intressant hat Anton seine Position und Positur nicht verändert. Die auf das erste Gemälde bezogene Stabilität des visuellen Fokus scheint mit der Stabilität seiner Position und Positur einherzugehen. Unmittelbar nach der Evaluation lockert Anton seine parallele Fußstellung, setzt den rechten $\mathrm{Fu} ß$ nach rechts und dreht auch den Körper leicht nach rechts. Im Dokument der Actionkamera wird diese Bewegung in einer leichten Veränderung des rechtsseitigen Bildausschnittes und einer leicht verschwommenen Qualität des Bildes sichtbar [Bild 7].

Anton geht einige Schritte im Mittelgang in Richtung Altar, wobei sein Blick weiterhin eine deutliche Linksorientierung besitzt. Anton thematisiert das zweite Gemälde nochmals eigenständig (noch eins), was aufgrund der Eingangsformulierung (riesige bilder) in dieser additiven Struktur (noch eins) nicht nötig wäre. 

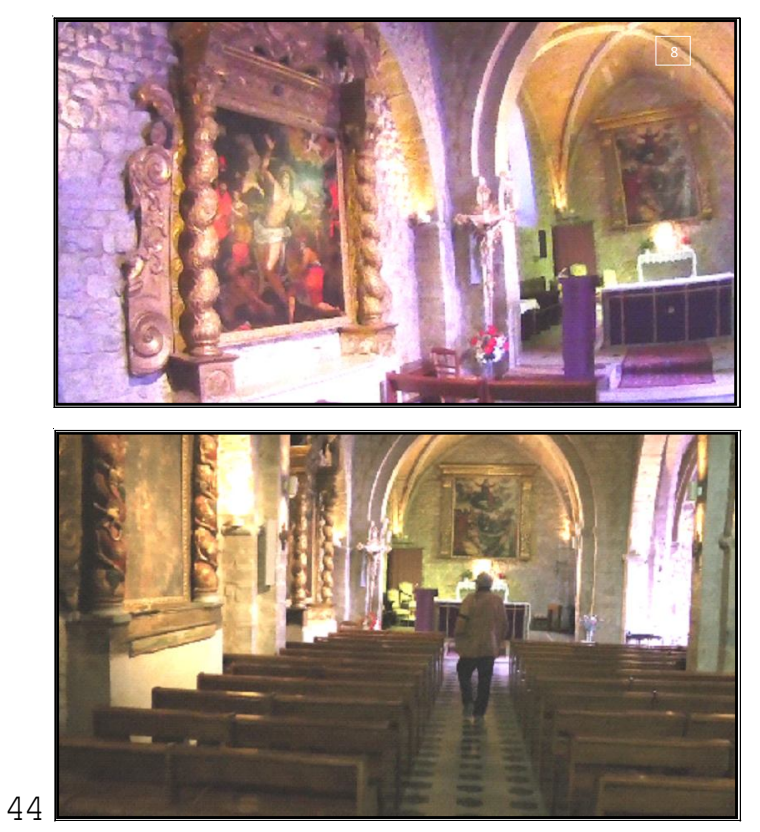

45

noch eins

$46 \quad(2.0)$

Die Thematisierung erfolgt noch während der Annäherung an das Gemälde. Im rechten Teil des Standbildes [Bild 8] ist zu sehen, dass Anton noch im Gehen begriffen ist und seinen Körper und Kopf erst langsam auf das zweite Bild ausrichtet.

Es scheint so zu sein, dass die interne Objektstruktur des eingangs konstituierten Segments „Wand“ oder „Wandabschnitt“ (ab da, wo ,zugemauert“ ist) jeweils eigenständig formuliert werden muss. Die segmentale Perspektive scheint also für die Objektkonstitution primär als Relevanz- oder Bezugsrahmen funktional zu sein, durch den zusammengehörige Objekte erfasst werden. Die segmental gerahmten Objekte werden dann - visuell und sprachlich - als eigenständige Foki behandelt.

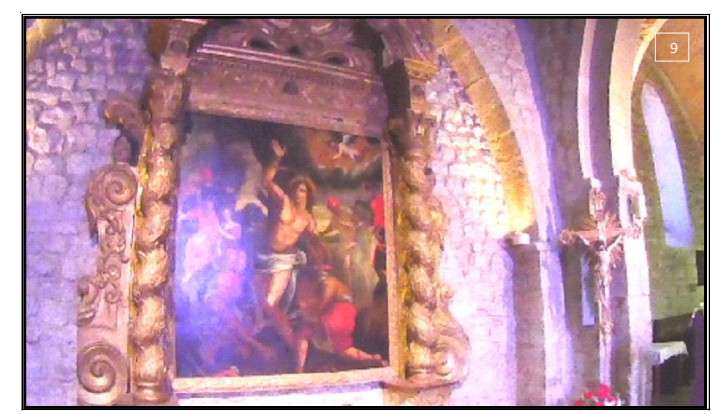

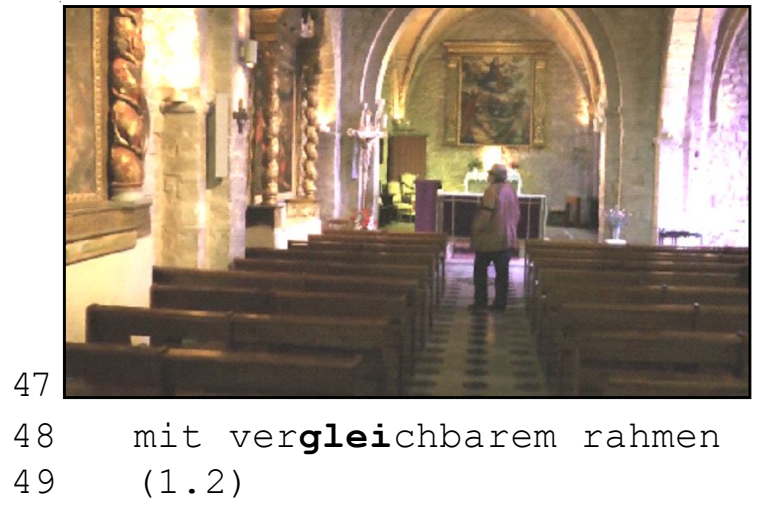

Das zweite Gemälde wird kontrastiv zum ersten thematisiert, was wohl dazu führt, dass auch bei diesem Gemälde gleich der Rahmen zur Sprache kommt. Der Vergleich als relevanter Aspekt der Objektkonstitution wird selbst als wichtiger Bestandteil der Thematisierung sprachlich repräsentiert (mit vergleichbarem rahmen). Der Vergleich führt dann zunächst zum Erkennen von Unterschieden der Rahmengestaltung (aber doch anders gestaltet). Anton hat sich inzwischen durch eine leichte Linksdrehung seines Körpers deutlicher auf das betrachtete Gemälde ausgerichtet [Bild 9]. Auch die Stellung seiner Füße hat sich als Bestandteil dieser Orientierung verändert, und sein linkes Bein ist nun nicht mehr in Bewegung.
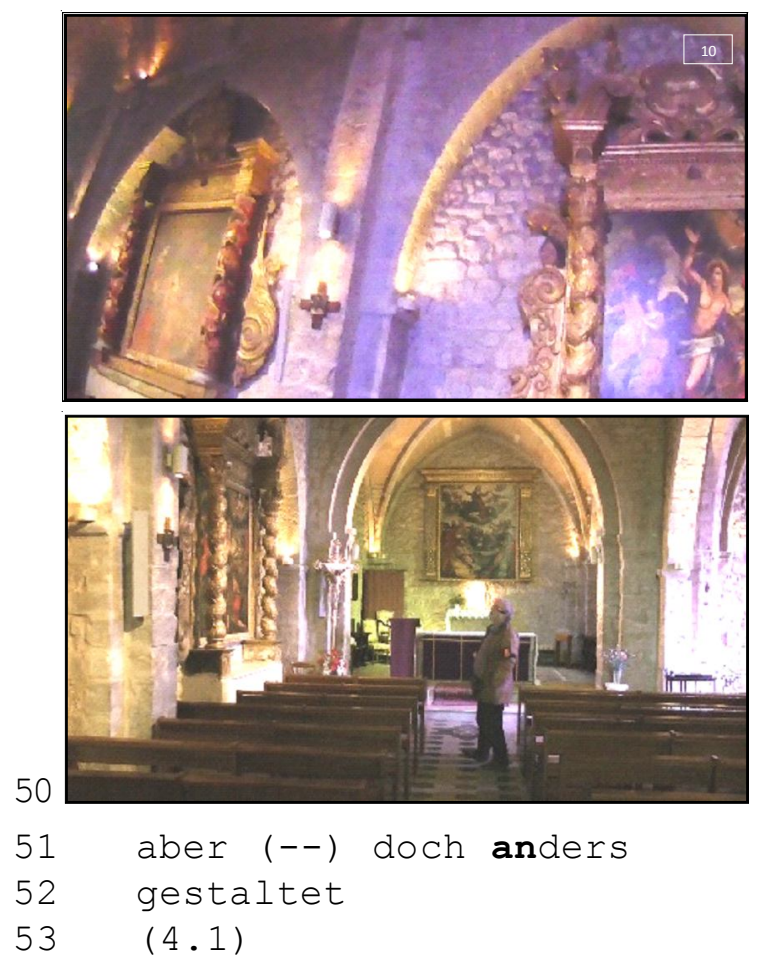
Die Vergleichsperspektive drückt sich auch in einer blicklichen Rückkehr zum ersten Gemälde aus. Antons Blick wandert zum ersten Gemälde und ruht dort für einen kurzen Moment. Gleichzeitig sagt er: aber (--) doch anders gestaltet. Die Actionkamera produziert genau zu dem Zeitpunkt ein klares, ruhiges Bild, als Anton mit anders die Vergleichsperspektive explizit thematisiert [Bild 10]. Sein Blick geht nach oben, und sein Körper wird zu diesem Zeitpunkt im Bild der Kontextkamera in einer deutlichen Streckung sichtbar. Dabei legt er seinen Kopf leicht in den Nacken.
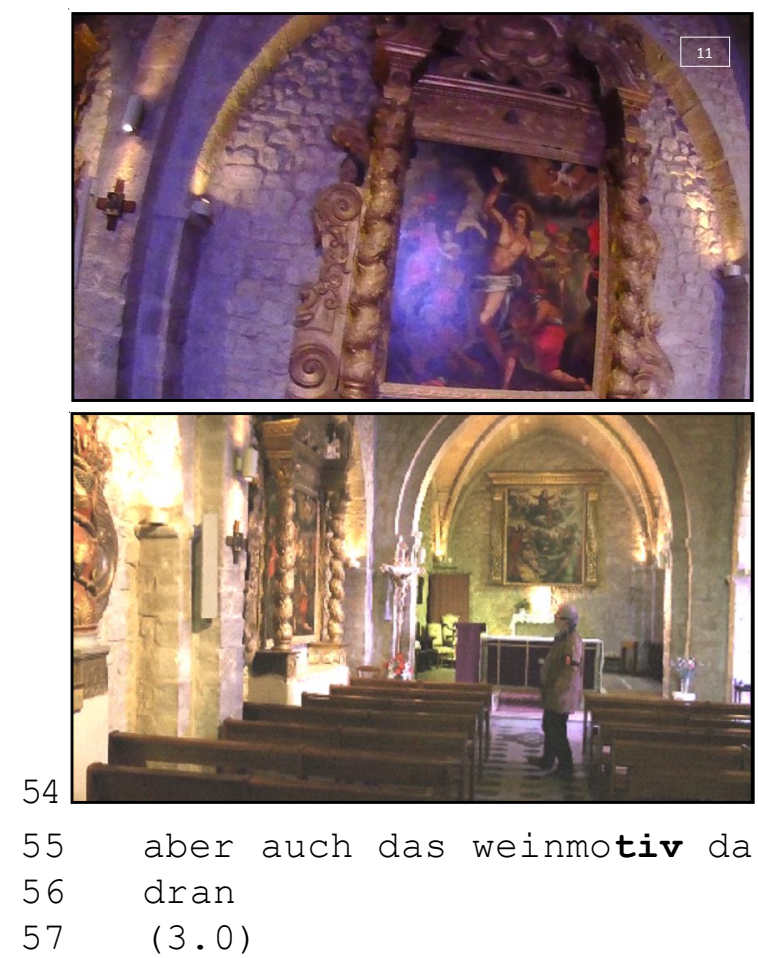

Nach einer kurzen Thematisierungspause erfolgt dann der Hinweis aber auch das weinmotiv da dran. Dieser Aspekt wurde bereits beim ersten Bild als relevantes Rahmendetail betont. In dem da dran versteckt sich als impliziter Referent der Rahmen des Gemäldes, mit dem sich Anton kontrastiv auseinandersetzt.

Die strukturierende Kraft der Kontrastierung führt insgesamt dazu, dass beim zweiten Gemälde nur diejenigen Aspekte thematisiert werden, die bereits beim ersten Bild zur Sprache gekommen sind. Bei der zweiten Objektkonstitution scheint als Prinzip so etwas wie eine Aspektübertragung die zentrale Rolle zu spielen. Außer einer Drehung des Kopfes nach rechts haben sich seine Körperpositur, sein Stand und die Stellung der Füße nicht verändert [Bild 11].
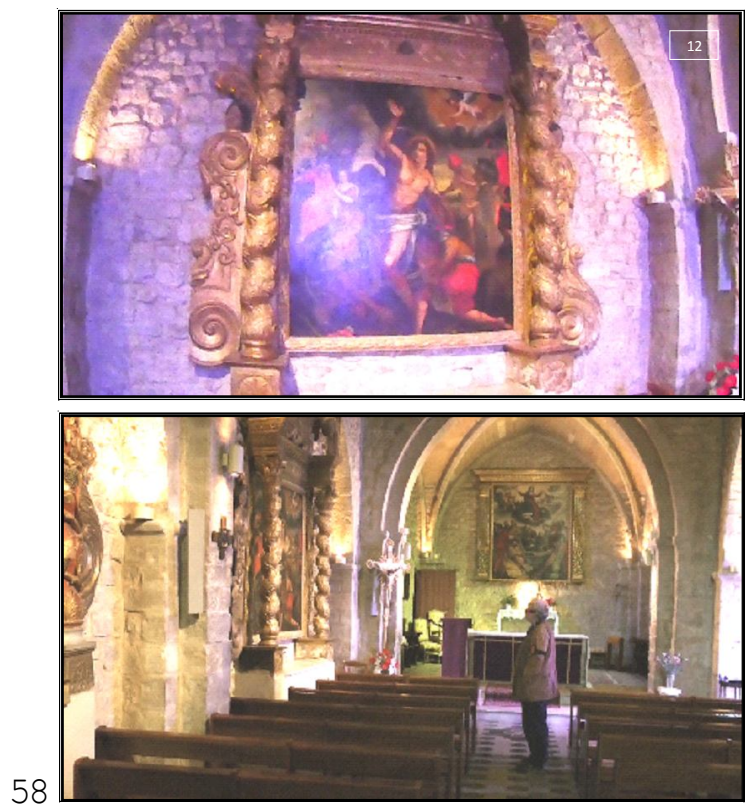

59 aber das bild (...)

Anton schließt auch die Beschäftigung mit dem zweiten Bild mit einer Evaluation ab, die nunmehr negativ ausfällt: aber das bild (...) das ist wohl nicht so: toll. Dieses Mal ist der Referent eindeutig (das bild), und dieser wird mit aber dem Rahmen, der bereits beim ersten Bild positiv evaluiert wurde (toll), gegenübergestellt. Der Rahmen des zweiten Bildes partizipiert also an der Positivevaluation des Rahmens vom ersten Gemälde. Wir können also erschließen, dass die Negativevaluation dem Gemälde, das heißt: der gestalteten Szene gilt.

Dabei ist sein Blick etwas nach unten gewandert, und sein Kopf liegt nur noch leicht im Nacken. Die deutlichste Veränderung seiner Positur sind die nun parallel verankerten Füße und die durchgestreckten Knie [Bild 12].

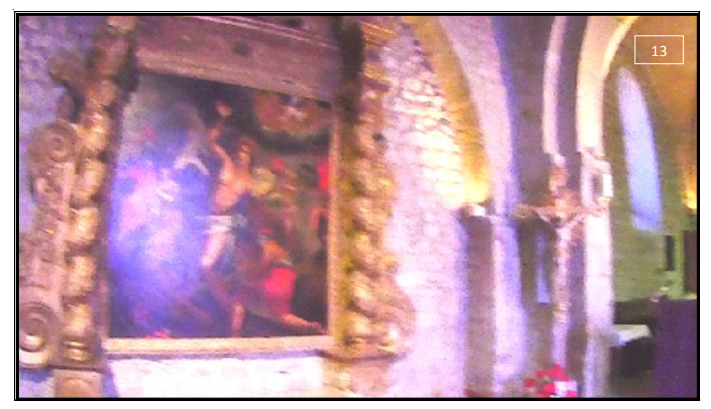




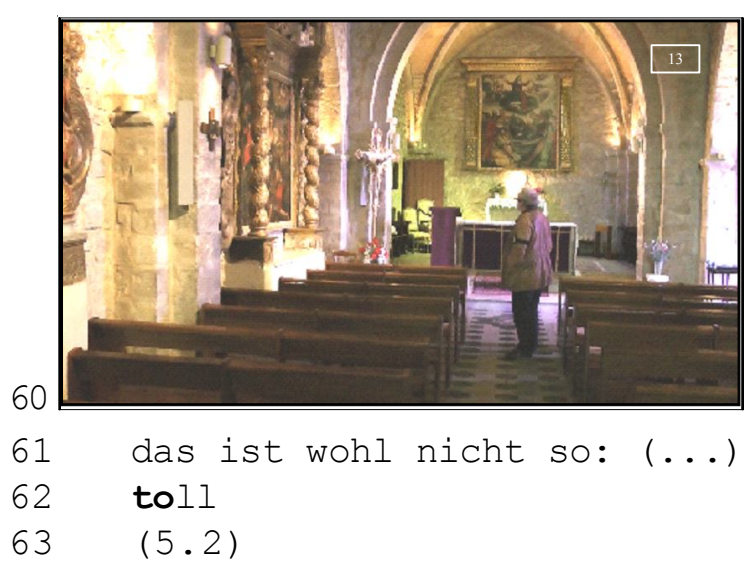

Interessant ist, dass wir darüber, was auf dem Gemälde dargestellt und gestaltet ist, nichts erfahren. Das gilt rückblickend auch für das erste Gemälde: Die dargestellten Inhalte beider Gemälde finden keinen Eingang in die Exothese. Die Rahmen der Gemälde scheinen sich der visuellen Wahrnehmung und der verbalen Thematisierung erfolgreich aufzudrängen, wohingegen die gestalteten Szenen der Gemälde abgewählt werden. Eine Motivierung hierfür kann in dem spezifischen Verhältnis von Bild und Rahmen bestehen, das nicht den Erwartungen des Betrachters entspricht. Die Rahmen sind gemessen an den Bildern - so groß und dominant, dass sie sich sowohl der visuellen Wahrnehmung als auch der Exothese aufdrängen - und für die gestalteten Szenen dann keinen Platz mehr lassen. Der Abschluss seiner Äußerung erfolgt simultan mit der beginnenden Auflösung seiner stabilen Verankerung. Sein Körper ist bereits weiter nach rechts gedreht, das Körpergewicht ruht auf dem rechten Bein, und das linke ist bereits ein wenig angehoben [Bild 13]. In der nachfolgenden fünfsekündigen Sprechpause (5.2) bewegt sich Anton im Mittelgang weiter auf den Altarraum zu.

\subsubsection{Fallanalytisches Resümee.}

Ordnet man die wichtigsten Punkte der zurückliegenden Analyse, dann wird deutlich: Anton konstituiert die beiden Gemälde - bezogen auf seine exothetischen Aktivitäten - primär als Bilderrahmen. Als einzigen Verweis auf die dargestellten Inhalte gibt es beim zweiten Gemälde nur eine Negativevaluation ohne Beschreibung. Weiterhin sind für die visuelle und exothetische Objektkonstitution die nachfolgenden Punkte relevant:

a. eine segmentale, auf architektonische Strukturen bezogene Primärperspektive,

b. das konstituierte Segment als Relevanzrahmen für die weitere Selektion von Gegenständen für die Objektkonstitution, c. eine Relevanzübertragung bei der Beschäftigung mit dem zweiten Gemälde, bei der nur die beim ersten Bild bereits thematisierten Aspekte wieder aufgegriffen werden,

d. eine insgesamt große Selektivität bei der Thematisierung des visuell Wahrgenommenen,

e. eine Synchronität von visueller Fokussierung und sprachlichem Format (enger visueller Fokus $=>$ extrem reduziertes Format $=>$ Einwortäußerung) und

f. die fehlende Thematisierung der dargestellten Inhalte der Gemälde.

\section{Objektkonstitution und Kirchenbesichtigung}

Die zurückliegende Analyse hat verdeutlicht, was wir unter Objektkonstitution verstehen: Antons Behandlung der „Gemälde als Bilderrahmen" ist eine eigenständige, aktive Konstitution der Bilder als relevante Objekte seiner Kirchenbesichtigung.

Konzeptionell betrachtet ist Objektkonstitution eine in situ bei der Auseinandersetzung mit dem objektiven Wahrnehmungsangebot des Raums von den Betrachtern erbrachte und auf mitgebrachten Relevanzen basierende aktive Neuschöpfung der räumlichen Strukturen eines Raums (hier: Kircheninnenraum). Die Spezifik der Objektkonstitution führt dazu, dass Anton, Saskia und Aurelia denselben Kirchenraum bei ihrer Besichtigung jeweils als etwas ganz Eigenständiges und Neues entwerfen. Dies geschieht einerseits durch die relevanzgeleitete Auswahl und andererseits durch die Spezifik der visuellen und verbalen Fokussierung der Objekte. Beides ist Ausdruck ihres jeweils zugrundeliegenden Besichtigungskonzeptes.

Anton realisiert ein Konzept, das primär auf architektonische und kunstgeschichtliche Aspekte des Raums und auf das Raumganze bezogen ist. Auf Grundlage dieser Orientierung gliedert bzw. segmentiert er den Raum und schafft dadurch einen Relevanzrahmen für selektive visuelle Wahrnehmung und wahrnehmungsbegleitende Kommentare, Deskriptionen und Evaluationen.

Saskia rekonstruiert den Kirchenraum primär als Manifestation und Symbol des christlichen Glaubens, repräsentiert durch die Person Jesus Christus. Sie ist die Einzige, die auf 
die dargestellte Thematik der beiden Gemälde eingeht, und diese in Zusammenhang mit dem Leben Jesu thematisiert (jesus als kind mit maria und josef denke ich, jesus kurz vor der kreuzigung).

Die Orientierung auf Jesus Christus stiftet bei ihr eine Kohärenz, die bei der Objektkonstitution über Objektgrenzen hinweggeht. Saskia thematisiert nach den beiden Gemälden auch das an der Grenze zum Altar angebrachte Kruzifix mit den Worten und hier als gekreuzigter mit plastikblumen. Sie stellt das Kruzifix als eine weitere Form der Darstellung Christus in einen unmittelbaren, sequenziell geordneten Zusammenhang mit den beiden Darstellungen der Gemälde. Das Kruzifix wird also nicht als isoliertes, eigenständiges Objekt genannt, sondern als weitere Darstellung im Kontext der beiden vorher betrachteten Gemälde, die chronologisch anschließt.

Den Abschluss dieser auf Christus bezogenen Orientierung stellt die Thematisierung des Altargemäldes dar. Saskia ist die Einzige, die diesem Gemälde überhaupt verbale Beachtung schenkt.

Aurelia wiederum konstituiert den Kirchenraum primär als für den Vollzug von Gottesdienst gebauten und ausgestatteten Handlungsrahmen. Sie organisiert ihre Besichtigung unter Bezug auf bestimmte, für den Gottesdienstvollzug relevante Aktivitätszusammenhänge. Sie fragt sich beispielsweise, wie man in der Kirche Orgel spielt, wenn man so sitzt, wie der Organist sitzen muss; wo man die Kerzen, die man vorne im Eingangsbereich kaufen kann, anzünden kann; ob es sich bei einem Objekt um ein weiteres Weihwasserbecken oder aber um ein Taufbecken handelt; dass man in den Kirchenbänken knien kann etc.

Für Aurelias handlungsfunktionale Raumperspektive spielt die Eigenart der speziell dafür gebauten Architektur (Wände, Gewölbe, Säulen, Schiffe), wie sie für Anton wahrnehmungsund thematisierungsleitend sind, nur eine untergeordnete Rolle. Unter dieser Perspektive konvergieren Beobachtungen, die für sich genommen zunächst merkwürdig erscheinen. Dies gilt beispielsweise für die wiederholte Thematisierung der Kirchenbänke und der mit ihnen verbundenen Möglichkeit, sich hinzuknien. Die Kirchenbänke als Hinweis auf den Vollzug von Gottesdienst finden (trotz ihrer raumausfüllenden Präsenz) weder bei Anton noch bei Saskia Erwähnung.

Mit ihrer Rekonstruktion des Raums als architektonische Voraussetzung des Vollzugs von Gottesdienst ist sie nahe an der konzeptionellen Vorstellung von Funktionsräumen als gebaute Lösungen für rekurrente interaktive Probleme, wie sie beispielsweise für die Konzeption "Interaktionsarchitektur" charakteristisch ist [Hausendorf, Schmitt, 2013, 2016].

\section{Schlussbemerkung und Ausblick}

Abschließend wollen wir uns noch einer Frage zuwenden, die relevant wird, wenn man weiter in Richtung, Objektkonstitution im Kontext von Kirchenbesichtigungen“" arbeiten will. Diese Frage lautet: Warum konstituieren alle drei Personen die Bilder überhaupt als relevante Objekte ihrer Kirchenbesichtigung?

Es scheint eine Qualität von Objekten zu geben, die weitgehend unabhängig vom jeweiligen Besichtigungskonzept der Kirchenbesichtiger eine starke Wahrnehmungsrelevanz entfalten. Diese Objekte fordern dazu auf, sich mit ihnen zu beschäftigen. Und es scheint nicht ganz einfach zu sein, diese Aufforderung zu ignorieren. Eine solche Aufforderungsqualität haben bei Weitem nicht alle Objekte im Kirchenraum.

Die Beantwortung der Frage, welche Objekte sich einer Wahrnehmung und Thematisierung besonders aufdrängen, kann nicht mit den Eigenschaften dieser Objekte alleine beantwortet werden. Vielmehr muss man von einem Spannungsverhältnis von Objekteigenschaften, einer spezifischen architektonischen Umgebung und mitgebrachten Erwartungen ausgehen.

Anton hatte aufgrund seines Eindrucks der Kirche von außen mit einem wesentlich größeren Innenraum gerechnet (hätt ich mir von außen größer vorgestellt). Er startet seine Besichtigung also mit einem Erwartungsbruch, der sich auch noch auf seine Wahrnehmung der Bilder auswirkt. Diese empfindet er - angesichts des kleinen Innenraums - als riesig.

Es ist letztlich die Disproportionalität der für den kleinen Innenraum der Kirche - viel zu großen Bilder, die sie für Anton auffällig und wahrnehmungs- und thematisierungsrelevant 
macht. In der Wahrnehmung und Thematisierung der Bilder zeigt sich darüber hinaus ein Aspekt, der auch seiner Wahrnehmung und Thematisierung des Kirchenraums insgesamt zugrunde liegt: eine starke Proportionalitäts- bzw. Symmetrieerwartung. So wie das nicht vorhandene (aber als normative Erwartung präsente) Linksschiff zur expliziten Beschäftigung auffordert, so motiviert auch eine normative Erwartung den Umgang mit den Bildern. Das Verhältnis der dargestellten Szenen zu den Gemälderahmen, die Proportionalität zur Wand, an der die Bilder hängen, und die Größe der Bilder relativ zu dem kleinen Raum führen letztlich zu einer expliziten Fokussierung und Bearbeitung.

Jenseits dieser fallspezifischen Aspekte wäre auf der Grundlage weiterer Analysen zu klären, welche Objekteigenschaften es denn genau sind, die im Zusammenspiel mit der Architektur des kirchlichen Innenraums und den mitgebrachten Erwartungen Wahrnehmungs- und Thematisierungsrelevanzen produzieren. Dabei wäre im Sinne der Vorstellung einer „relevanten Abwesenheit" auch danach zu fragen, ob es im Kirchenraum Objekte gibt, die keine Relevanz im Hinblick auf objektkonstituierende Aktivitäten entfalten und in systematischer Weise übergangen werden.

\section{ANMERKUNGEN}

${ }^{1}$ Alle Namen sind maskiert.

${ }^{2}$ Die nachfolgende Liste erhebt keinen Anspruch auf Vollständigkeit. Sie soll lediglich die konzeptionelle Breite für das oben skizzierte „Vermittlungsproblem“"verdeutlichen.

${ }^{3}$ Siehe dazu beispielsweise [Lynch, 2002; Mondada, 2012; Sacks, Schegloff, Jefferson 1974; Speer, 2002].

${ }^{4}$ Zum Beobachterparadoxon siehe beispielsweise [Labov, 1972] und [Burdick, 2008].

${ }^{5}$ So betrachtet Anton zu einem späteren Zeitpunkt (siehe Seite 25) ein im Altarvorraum angebrachtes Kruzifix längere Zeit und sagt dazu keine kanzel.

${ }^{6}$ Von den drei Bildbetrachtungen, die von verschiedenen Personen in der Kirche von Gréoux les Bains dokumentiert und analysiert wurden, kann aus Platzgründen nur ein Fall exemplarisch präsentiert werden. In der Schlussbemerkung werde ich jedoch kurz auf zwei weitere Besichtigungskonzepte eingehen, die von Saskia und Aurelia beim Besuch derselben Kirche realisiert wurden.
${ }^{7}$ Die Transkription erfolgt in Anlehnung an GAT 2 [Gesprächsanalytisches Transkriptionssystem, 2009].

${ }^{8}$ Die Zuordnung der Abbildungen zum Transkript wird durch Fett-Markierungen im Transkript sichtbar gemacht. Die zwei übereinanderstehenden Standbilder geben simultan die Perspektive der Actionkamera (oben) und der Kontextkamera (unten) zum Zeitpunkt der fett markierten Äußerung im Transkript wieder. Die beiden Standbilder werden daher immer gemeinsam als ein Bild nummeriert. Das untere Bild wird nur dann eigenständig nummeriert, wenn beide Standbilder beim Seitenumbruch getrennt werden.

${ }^{9}$ In der Konversationsanalyse (beispielsweise [Mondada, 2016]) hat sich zur Kennzeichnung unterschiedlicher Versuche, neben der schriftlichen Notation des Gesprochenen auch Abbildungen, Standbilder, Markierungen und andere nichtschriftliche Dokumentationsformen im Transkript zu integrieren, die Bezeichnung „multimodales Transkript" eingebürgert. Diese Bezeichnung ist jedoch irreführend, denn solche Transkripte sind selbst nicht multimodal. Korrekt müsste es „multimediales Transkript“"heißen. Zumindest, wenn man unter Multimodalität die Gesamtheit der den Menschen in der Interaktion zur Verfügung stehenden Ausdrucksressourcen und damit eine konstitutive Eigenschaft von Interaktion selbst meint. Solche Transkripte arbeiten selbst nicht mit unterschiedlichen Modalitäten im interaktionskonstitutiven Sinne, sondern nutzen unterschiedliche Medien (Schrift, Bild, Markierungen etc.), um Multimodalität abzubilden.

${ }^{10}$ Würden die beiden Gemälde im Kontext großformatiger Exponate in einer Ausstellung oder einem Museum hängen, würde ihre objektive Größe sicherlich ebenfalls keinen Grund für eine Thematisierung abgeben.

\section{LITERATURLISTE}

Bergmann J.R., Luckmann T. Reconstructive genres in everyday communication. Quasthoff U., ed. Aspects of oral communication. Berlin; New York, De Gruyter, 1995, pp. 289-304.

Burdick C. Das Beobachterparadoxon in der Feldarbeit: Eine linguistische Perspektive [The observer's paradox in fieldwork: a linguistic perspective]. Saarbrücken, VDM Verlag Dr. Müller, 2008. $80 \mathrm{~S}$.

Deppermann A. How does 'cognition' matter to the analysis of talk-in-interaction? Language Sciences, 2012, vol. 34, pp. 746-767.

Deppermann A., Feilke H., Linke A. Sprachliche und kommunikative Praktiken: Eine Annäherung aus 
linguistischer Sicht [Linguistic and communicative practices: an approach from a linguistic perspective]. Deppermann A., Feilke H., Linke A., eds. Sprachliche und kommunikative Praktiken [Verbal and communicative practices]. Berlin; Boston, De Gruyter, 2016, pp. 1-23 (Jahrbuch des Instituts für Deutsche Sprache 2015).

Ehlich K., Rehbein J. Sprachliche Handlungsmuster [Linguistic action types]. Hg. Soeffner H.-G. Interpretative Verfahren der Sozial- und Textwissenschaften [Interpretative methods in social and textual sciences]. Stuttgart, Metzlersche Verlagsbuchhandlung, 1979, pp. 243-274.

Ehlich K., Rehbein J. Zur Konstitution pragmatischer Einheiten in einer Institution: Das Speiserestaurant [The constitution of pragmatic units within an institution: the restaurant]. Hg. Wunderlich D. Linguistische Pragmatik [Linguistic Pragmatics]. Frankfurt a. M., Akademische Verlagsgesellschaft Athenaion, 1972, pp. 209-254.

Eigenschaften gesprochener Sprache [Characteristics of spoken language]. Fiehler R., Barden B., Elstermann M., Kraft B. Tübingen, Narr, 2004,pp. 99-110.

Garfinkel H. Studies in ethnomethodology. Englewood Cliffs, NY, Prentice Hall Inc., 1967. 288 p.

Gesprächsanalytisches Transkriptionssystem 2 (GAT 2) [A system for transcribing conversation 2 (GAT2)]. Selting M., Auer P., Barth-Weingarten D., Bergmann J. et al. Gesprächsforschung - OnlineZeitschrift zur verbalen Interaktion [Conversation analysis - online journal for the study of verbal interaction], 2009, pp. 353-402. Available at: http:// www.gespraechsforschung-online.de/fileadmin/ dateien/heft2009/px-gat2.pdf. Abrufdatum: 28.02.2017.

Göpferich S. Praktische Handreichung für Studien mit lautem Denken und Translog [Practical assistance for the study of thinking aloud and Translog]. (2000 und 2006). Institut für Theoretische und Angewandte Translationswissenschaft (ITAT). Karl-Franzens-Universität Graz, 2007. Available at: http://www.susanne-goepferich.de/Handreichung.pdf. Abrufdatum: 18.10.2014.

Günthner S. Gattungen in der sozialen Praxis. Die Analyse, kommunikativer Gattungen “ als Textsorten mündlicher Kommunikation [Genres within social praxis: Analyzing communicative genres as text types of verbal communication]. Deutsche Sprache [German Language], 1995, iss. 3, pp. 193-218.

Günthner S. Von Konstruktionen $\mathrm{zu}$ kommunikativen Gattungen: Die Relevanz sedimentierter Muster für die Ausführung kommunikativer Aufgaben [From constructions to communicative genres: the relevance of sedimented types for the accomplishment of communicative tasks]. Deutsche Sprache [German Language], 2006, iss. 34, no. 1/2, pp. 173-190.
Günthner S., Knoblauch H. Culturally patterned speaking practices. The analysis of communicative genres. Pragmatics, 1994, iss. 5, no. 1, pp. 1-32.

HausendorfH., Schmitt R. Interaktionsarchitektur und Sozialtopographie. Eine Konzeptklärung [Architecture-for-interaction and social topography: a conceptual clarification]. Hrsg. HausendorfH., Schmitt R., Kesselheim W. Interaktionsarchitektur, Sozialtopographie und Interaktionsraum [Architecture-for-interaction, social topography, and interaction space]. Tübingen, Narr, 2016, pp. 27-54.

HausendorfH., Schmitt R. Interaktionsarchitektur und Sozialtopographie. Umrisse einer raumlinguistischen Programmatik [Architecture-for-interaction and social topography: programmatic outlines of a linguistic perspective on space]. Arbeitspapiere des UFSP Sprache und Raum (SpuR) [Working papers of the URPP Language and Space (SpuR)], 2013, no. 01, S. 59. Available at: http://www.zora.uzh.ch/78153/1/ SpuR_Arbeitspapiere_Nr01_Mai2013.pdf.Abrufdatum: 28.02.2017.

Heidtmann D. Multimodalität der Kooperation im Lehr-Lern-Diskurs: Wie Ideen für Filme entstehen [The multimodality of teacher-learner discourse. How ideas for movies are developed]. Studien zur Deutschen Sprache. Bd. 50 [Studies on German Language. Vol. 50]. Tübingen, Narr Francke Attempto Verlag, 2009. $340 \mathrm{~S}$.

Heine L. Introspektion [Introspection]. Einführung in empirische Forschungsmethoden für Deutsch als Fremd-und Zweitsprache [Introduction to empirical research methods of German as a foreign and second language]. Hrsg. Settinieri J., Demirkaya, S., Gültekin-Karacoc, N. Paderborn, Schöningh UTB, 2014, pp. 123-135.

Heine L., Schramm K. Lautes Denken in der Fremdsprachenforschung: Eine Handreichung für die empirische Praxis [The concept of thinking aloud in foreign language research: Practical recommendations]. Hg. Vollmer H.J. Synergieeffekte in der Fremdsprachenforschung. Empirische Zugänge, Probleme, Ergebnisse [Synergetic effects in the study of foreign languages. Empirical approaches, problems, results]. Frankfurt am Main, Peter Lang Verlag, 2007, pp. 167-206.

Hester S., Francis D. Analyzing visually available mundane order: A walk to the supermarket. Visual Studies, 2003, iss. 18, no. 1, pp. 36-46.

Kallmeyer W. Beraten und Betreuen. Zur gesprächsanalytischen Untersuchung von helfenden Interaktionen [Counceling and mentoring. Talk-ininteraction research of supportive interaction]. Zeitschrift für qualitative Bildungs-, Beratungs-, und Sozialforschung [Journal for the qualitative study of education, counseling and social relations], 2000, iss. 2, pp. 227-252. 
Kallmeyer W. Handlungskonstitution im Gespräch. Dupont und sein Experte führen ein Beratungsgespräch [Action constitution in conversation. Dupont and his expert engage in a consultation]. Hrsg. Gülich E., Kotschi T. Grammatik, Konversation, Interaktion. [Grammar, conversation, interaction]. Berlin, De Gruyter, 1985, pp. 81-122.

Kallmeyer W., Schütze F. Konversationsanalyse [Conversation analysis]. Studium Linguistik [The study of linguistics], 1976, pp. 1-28.

Kallmeyer W., Schütze F. Zur Konstitution von Kommunikationsschemata der Sachverhaltsdarstellung [On the constitution of communicative scripts for the presentation of facts]. Hg. Wegner D. Gesprächsanalysen. Vorträge gehalten anlässlich des 5. Kolloquiums des Instituts für Kommunikationsforschung und Phonetik (Bonn, 1416 Oktober 1976) [Conversation analysis. Lectures held at the 5th colloquium of the Institute for Communication research and Phonetics (Bonn, October 14th-16th, 1976)]. Hamburg, Buske, 1977, pp. 159-274.

Klemm M. Sprechhandlungsmuster [Patterns of verbal action]. Hrsg. Holly W., Püschel U., Bergmann J. Der sprechende Zuschauer. Wie wir uns Fernsehen kommunikativ aneignen [The talking spectator. How we adopt television communicatively]. Opladen, Verlag für Sozialwissenschaften, 2001, pp. 83-114.

Konrad K. Lautes Denken [Thinking aloud]. Hrsg. Mey G., Mruck K. Handbuch Qualitative Forschung in der Psychologie [Handbook of qualitative studies in psychology]. Wiesbaden, VS Verlag, 2010, pp. 476-490.

Labov W. Sociolinguistic patterns. Philadelphia, University of Philadelphia, 1972. 364 S.

Levinson S.C. Activity types and language. Linguistics, 1979, iss. 17, no. 5-6, pp. 2-100.

Levinson S.C. Activity types and language. Drew P., Heritage J., eds. Talk at work: Interaction in institutional settings. Cambridge, Cambridge University Press, 1992, pp. 66-100.

Luckmann T. Grundformen der gesellschaftlichen Vermittlung des Wissens: Kommunikative Gattungen [Basic features of the societal transmission of knowledge: communicative genres]. Kölner Zeitschrift für Soziologie und Sozialpsychologie, Sonderheft [Cologne journal for sociology and social psychology, special issue], 1986, vol. 27, pp. 191-211.

Luckmann T. Kommunikative Gattungen im kommunikativen Haushalt einer Gesellschaft [Communicative genres in a society's communicative household]. Hg. Smolka-Koerdt et al. Der Ursprung von Literatur [The origin of literature]. München, Fink, 1988, pp. 279-288.

Lynch M. From naturally occurring data to naturally organized ordinary activities: comment on Speer. Discourse Studies, 2002, vol. 4, pp. 531-537.
Mondada L. The conversation analytic approach to data collection. Sidnell J., Stivers T., eds. The handbook of conversation analysis. London, Blackwell-Wiley, 2012, pp. 32-56.

Mondada L. Zwischen Text und Bild: Multimodale Transkription [Between text and picture: multimodal transcription]. Studien zur Deutschen Sprache [Studies on German Language], 2016, vol. 72, pp. 111-160.

Pache I. Gefährdete Reziprozität. Kommunikative Praktiken im Bewerbungsgespräch [Endangered reciprocity. Communicative practices in the interview]. Springer eBook (Reihe Sprachwissenschaft), 2004. Available at: https://archive.org/stream/springer 10.1007-978-3-322-81317-6/10.1007-978-3-322-813176 djvu.txt. Abrufdatum: 28.02.2017.

Sacks H., Schegloff, E.A., Jefferson G. A simplest systematics for the organization of turn-taking for conversation. Language, 1974, vol. 50, no. 4, part 1, pp. 696-735.

Schmitt R. Inszenieren: Struktur und Funktion eines gesprächsrhetorischen Verfahrens [Staging: Structure and function of a rethoric procedure in talk-in-interaction]. Gesprächsforschung. Online-Zeitschrift zur verbalen Interaktion [Conversation analysis - online journal for the study of verbal interaction], 2003, iss. 4, pp. 186-250. Available at: http://www.gespraechsforschungonline.de/fileadmin/dateien/heft2003/ga-schmitt.pdf. Abrufdatum:28.02.2017.

Schmitt R. Gehen als situierte Praktik: „Gemeinsam gehen“" und ,hinter jemandem herlaufen“ [Walking as situated practice: Walking together and going behind someone]. Gesprächsforschung OnlineZeitschrift zur verbalen Interaktion [Conversation analysis - online journal for the study of verbal interaction], 2012a, iss. 13, pp. 1-44. Available at: http:/ /www.gespraechsforschung-online.de/fileadmin/ dateien/heft2012/ga-schmitt.pdf. Abrufdatum: 20.02.2017.

Schmitt R. Störung und Reparatur eines religiösen Ritus: Die erloschene Osterkerze [Disruption and reparation of a religious ritual: the expired paschal candle]. Hrsg. Klein W., Habscheid S. Dinge und Maschinen in der Kommunikation. Sonderheft der Zeitschrift für Literaturwissenschaft und Linguistik [Objects and machines in interaction. Special issue, study of literature and linguistics], 2012b, no. 168, pp. 62-91.

Schreier M. Qualitatives Untersuchungsdesign [Qualitative research design]. Hg. Groeben N. Empirische Unterrichtsforschung in der Literaturund Lesedidaktik [Empirical studies on the didactics of literature and reading]. Weinheim; München, Iuventa Verlag, 2006, pp. 343-359.

Speer S.A. 'Natural' and 'contrived' data: a sustainable distinction? Discourse Studies, 2002, vol. 4, iss. 4 , pp. 511-525. 
Steen G.J. Lautes Denken zwischen Validität und Reliabilität [Thinking aloud between validity and reliability]. Hg. Barsch A. Empirische Literaturwissenschaft in der Diskussion [Discussion on empirical literary studies]. Frankfurt, Suhrkamp Taschenbuch Wissenschaft, 1994, pp. 297-305.
Studien zur Deutschen Sprache. Bd. 72: Interaktionsarchitektur, Sozialtopographie und Interaktionsraum [Studies on German Language. Vol. 72: Architecture-for-interaction, social topography, and interaction space]. Hrsg. Hausendorf H., Schmitt R., Kesselheim W. Tübingen, Narr Francke Attempto, 2016. $448 \mathrm{~S}$.

\section{Information about the Author}

Reinhold Schmitt, Doctor of Sociology, Member of the Department of Pragmatics, Institute for the German Language, Augustaanlage, 32, D-68165 Mannheim, Germany, reinhold.schmitt@idsmannheim.de, http://orcid.org/0000-0003-0981-8039.

\section{Информация об авторе}

Райнхольд Шмитт, доктор социологии, научный сотрудник отделения прагматики, Институт немецкого языка, ул. Аугустаанлаге, 32, D-68165 г. Мангейм, Германия, reinhold.schmitt@idsmannheim.de, http://orcid.org/0000-0003-0981-8039.

\section{Information über den Autor}

Reinhold Schmitt, Doktor der Soziologie, Mitarbeiter der Abteilung Pragmatik, Institut für Deutsche Sprache, Augustaanlage, 32, D-68165 Mannheim, Deutschland, reinhold.schmitt@ids-mannheim.de, http:// orcid.org/0000-0003-0981-8039. 